\title{
UAV-Enabled Mobile Edge-Computing for IoT Based on AI: A Comprehensive Review
}

\author{
Yassine Yazid ${ }^{1,2, *}$ Imad Ez-Zazi $^{3}{ }^{(1)}$, Antonio Guerrero-González $^{2}$, Ahmed El Oualkadi $^{1}$ (i) and Mounir Arioua ${ }^{1}$ \\ 1 Laboratory of Information and Communication Technologies (LabTIC), National School of Applied Sciences \\ of Tangier (ENSATg), Abdelmalk Essaadi University, ENSA Tanger, Route Ziaten, Tangier BP 1818, Morocco; \\ aeloualkadi@uae.ac.ma (A.E.O.); m.arioua@uae.ac.ma (M.A.) \\ 2 Department of Automation, Electrical Engineering and Electronic Technology, Universidad Politécnica de \\ Cartagena, Plaza del Hospital 1, 30202 Cartagena, Spain; antonio.guerrero@upct.es \\ 3 National School of Applied Sciences of Fez (ENSAF), Sidi Mohamed Ben Abdellah University, Fez BP 2626, \\ Morocco; imad.ezzazi@usmba.ac.ma \\ * Correspondence: y.yazid@uae.ac.ma
}

\section{check for}

updates

Citation: Yazid, Y.; Ez-Zazi, I.; Guerrero-González, A.; El Oualkadi, A.; Arioua, M. UAV-Enabled Mobile Edge-Computing for IoT Based on AI: A Comprehensive Review. Drones 2021, 5, 148. https://doi.org/ $10.3390 /$ drones5040148

Academic Editors: Vishal Sharma, Abderrahmane Lakas, Mohammed Atiquzzaman, Omar Sami Oubbati, Sahar Hoteit and Taieb Znati

Received: 27 October 2021

Accepted: 9 December 2021

Published: 13 December 2021

Publisher's Note: MDPI stays neutral with regard to jurisdictional claims in published maps and institutional affiliations.

Copyright: (c) 2021 by the authors. Licensee MDPI, Basel, Switzerland. This article is an open access article distributed under the terms and conditions of the Creative Commons Attribution (CC BY) license (https:// creativecommons.org/licenses/by/ $4.0 /)$.
Abstract: Unmanned aerial vehicles (UAVs) are becoming integrated into a wide range of modern IoT applications. The growing number of networked IoT devices generates a large amount of data. However, processing and memorizing this massive volume of data at local nodes have been deemed critical challenges, especially when using artificial intelligence (AI) systems to extract and exploit valuable information. In this context, mobile edge computing (MEC) has emerged as a way to bring cloud computing (CC) processes within reach of users, to address computation-intensive offloading and latency issues. This paper provides a comprehensive review of the most relevant research works related to UAV technology applications in terms of enabled or assisted MEC architectures. It details the utility of UAV-enabled MEC architecture regarding emerging IoT applications and the role of both deep learning (DL) and machine learning (ML) in meeting various limitations related to latency, task offloading, energy demand, and security. Furthermore, throughout this article, the reader gains an insight into the future of UAV-enabled MEC, the advantages and the critical challenges to be tackled when using AI.

Keywords: UAVs; IoT; cloud computing; edge computing; MEC; AI; review

\section{Introduction}

The Internet of Things (IoT) paradigm is constantly expanding to cover many fields and applications. IoT devices present many benefits for improving services in a broad range of contexts. The number of connected devices is forecasted to reach impressive heights in the coming years. According to CISCO, more than 70 billion devices will be connected by the end of 2025 [1,2]. Recently, unmanned aerial vehicles (UAVs), typically known as drones, have attracted considerable attention from academia and industrial communities due to their excellent capabilities and attractive features. They promise to bring an ideal contribution to the field of IoT, based on their unique characteristics. They offer elastic and flexible mobile features, as required in many omnipresent IoT applications, making their integration into wireless network communication easy and feasible.

Moreover, integrating embedded intelligent devices such as cameras and sensors on UAVs offer many advantages when monitoring hostile and unreachable environments. In addition, they can potentially provide many services in the context of modern IoT interconnection, such as smart cities, smart farming, smart factories, smart grids, and intelligent transportation. The development of communication protocols and networks has also improved the tracking and maneuverability of drones to cover many application requirements, as well as their contribution as a critical component of the communication network [3]. UAVs can also guarantee reliable connectivity in hostile areas with limited 
or unavailable communication infrastructure. Typically, UAVs can be deployed as aided communication networks by integrating them into the peripheral network as flying base stations (BSs) [4,5], relay nodes [6], or terminal nodes [7]. Therefore, they act primarily as a means of enabling flexible communication services in remote areas when interconnections with existing networks are lost. To this end, UAVs can be used in edge-computing (EC), fog-computing (FC), and as crucial equipment in cellular networks [8].

IoT networks enable comprehensive connectivity for many mobile users by enabling various emerged application services (e.g., automatic surveillance, unmanned control vehicles and object recognition). Centralized servers, such as cloud computing (CC) facilities and data centers, generally process and analyze the generated data by carrying out computational intelligence based on artificial intelligence (AI). These centralized systems are quick to deploy, inexpensive to maintain, and practical when data need to be centrally controlled. However, they are mostly constrained by their high failure rate, security risks and access delay. Data processing at the central level causes considerable time delays that affect the overall system quality of service (QoS), especially regarding the response-time requirement for latency-sensitive applications. Decentralized and distributed systems have emerged to solve the issue of centralized system constraints. The decentralized system relies on servers that are supposed to store a copy of the resources. Users in distributed systems have equal access to data, yet user rights can be enabled as necessary, and data ownership can be shared efficiently. Hardware and software resources are also allocated between users, increasing truthfulness, reducing component failure, and enhancing reliability.

EC has recently been introduced to provide intermediate devices between the data generator and CC services. This reduces latency and provides efficient bandwidth utilization. However, due to their limited computing power and battery life, mobile devices struggle to provide these services. To this end, mobile edge computing (MEC) solutions have been employed to ameliorate the intensive computation and offloading bottlenecks between end-nodes and centralized data servers [9]. They allow mobile devices to offload their computation duties onto servers at the edge of the IoT network, such as cellular BSs or access points. In contrast to traditional CC, MEC servers can be close to end-users. As a result, MEC servers can deliver low latency and secure services, while saving energy for mobile users.

Recently, UAVs have been integrated as air-ground equipment to fulfill the processing and storage needs at IoT network edges. As a promising solution, they could provide ultralow latency support for latency-sensitive applications, facilitate the distribution of big data processing and analysis, improve the scalability and management of massive mobile connectivity, as well as content caching and mobile delivery [10]. The advantage presented by their flexible mobility has facilitated the integration of UAVs with MEC services to provide UAV-enabled MEC networks. UAV-enabled MEC is typically deployed in unpredictable environments such as in wild, desert, and complex landscapes. Furthermore, the computation performance may be adapted based on the distance that UAVs can smoothly control. This combination of UAV technology with MEC makes the system more advantageous and flexible than traditional MEC systems, which are constrained by high deployment costs due to their fixed architecture. In addition, the integration of UAV-enabled MEC with the most recent security approaches, such as the blockchain structure, increases the security level [11]. The blockchain is a distributed ledger that gives a secure, immutable, transparent, and trustworthy environment for the UAVs to store their data as a transaction in a chain of blocks [12]. This is a viable solution to mitigate the security and privacy issues related to UAV networks. Therefore, integrating blockchain into UAVs ensures the MEC system's trustworthiness with the additional, advantageous features of automating data transaction, decision-making, storage, and verification [13]. Therefore, UAVs can play a crucial role in enabling MEC capability in IoT applications. However, many critical issues have yet to be addressed to improve the performance of UAV-enabled MEC. Most of the impairments are related to drones as they are autonomous and battery-powered, with limited resources. Furthermore, given the broadcast nature of communications between 
ground-based users and UAVs, many performance aspects should be addressed, including security, task offloading, energy consumption, resource allocation, computing capacity, and user latency performance under various channel circumstances.

Since this topic is revolutionary in the IoT research field, reviewing the current scientific achievements and technological limitations of UAV-enabled MEC networks is of paramount importance. Furthermore, the limits of existing surveys on UAV-enabled MEC in IoT applications have motivated us to put forward a detailed review. This paper explores the use of UAVs in emerging IoT applications and the utility of both deep learning (DL) and machine learning (ML) in UAV-enabled MEC systems. For instance, the authors of [14] have elaborated a review about the energy efficiency constraints related to UAV-enabled MEC systems for IoT devices. Additionally, the authors of $[15,16]$ have presented a survey about communication and networking technologies for UAVs. The research work presented in [17] has addressed both fundamental and recent advances of computation offloading in UAV-enabled MEC. However, only a few of the challenges are mentioned in the above works, including energy, communication facilities, and limited resource allocation issues. In this paper, the most critical issues and challenges of UAV-enabled MEC based on AI are accurately outlined and discussed.

Indeed, there are several recent reviews about the UAV-enabled MEC paradigm. However, most of the previous reviews have focused on UAV energy efficiency, communication technologies connecting various UAVs, and computation offloading in UAV-enabled MEC. Our review considers a more practical and recent scenario wherein massively generated data need to be processed and analyzed using AI algorithms for decision-making at the edge of networks. This has not been considered in the previous works. Adopting AI methods would affect all the requirements that have already been investigated, such as energy efficiency, communication technologies, processing capacity, privacy and security, and the autonomy of UAV-enabled MEC. Almost all domains are currently relying on AI to outperform the traditional systems and provide decision-making systems. For instance, Industry 4.0 has newly emerged as a revolutionary paradigm in which intelligent and autonomous drones based on AI would be the key element. However, the integration of autonomous UAVs based on AI is not obvious in most practical cases. This is why UAVenabled MEC based on AI would enable many applications in the industry community to deploy their solutions, by cooperatively executing and processing the massive volumes of confidential data near the users at the edge of the network. Consequently, the current review highlights the most recent challenges and open issues of UAV-enabled MEC based on AI, considering their various architectures and applications. We will discuss the most recent related works on data processing at the edge, based on AI, and their advantages and inconveniences regarding the UAV environment. We will even emphasize the effect of AI on energy efficiency, communication technologies, processing capacity, and security when UAV-enabled MEC architecture is considered. In addition, we propose a list of applications with open issues that have to be taken into account when considering a UAV-enabled MEC architecture.

The rest of this paper is structured as follows. Section 2 gives an overview of UAVs and highlights some of their related applications. Section 3 focuses on the possible opportunities enabled by UAVs, focusing on MEC servers. Section 4 highlights the UAV-enabled MEC based on AI. Section 5 lists significant issues and open research directions related to UAVenabled MEC systems. Finally, Section 6 presents a conclusion.

\section{UAV System Model}

Recently, there has been an increase in customer demand for airborne intelligence missions, which has led to the design and development of various types of UAVs in various shapes and flight modes. This section highlights the different styles and characteristics of UAVs and their application scenarios. 


\subsection{UAVs Classification}

Deploying a UAV network (known as swarms in IoT contexts) is becoming a practical and necessary requirement for many applications. Various UAVs are currently available on the market, with distinctive features such as supportable altitude, speed, and battery autonomy, for multiple applications. Generally, the selection of any UAV model should fulfill many of the criteria of the targeted application, such as QoS, energy capacity, environmental restrictions, and federal rules [18]. There are three types of UAVs: low-altitude platforms (LAPs), high-altitude platforms (HAPs), and satellites. Since this work focuses on UAV-enabled MEC, UAVs are described based on many aspects.

Figure 1 categorizes UAVs into the LAPs category based on their type, size, range, and type of rotors. Figure 2 shows the shape and form of three kinds of commercialized UAVs: a rotary-wing drone (Figure 2a), a fixed-wing drone (Figure $2 \mathrm{~b}$ ), and a fixed wing hybrid VTOL UAV (Figure 2c).

\section{LAP UAV classification}

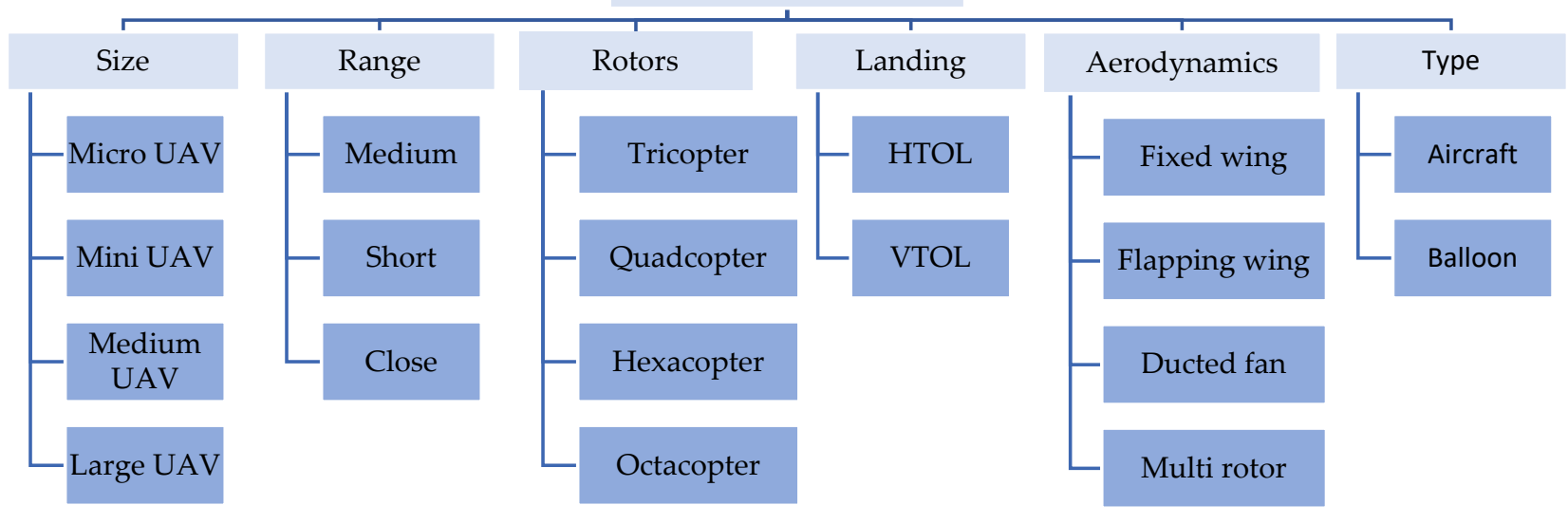

Figure 1. Classification of LAP UAVs.

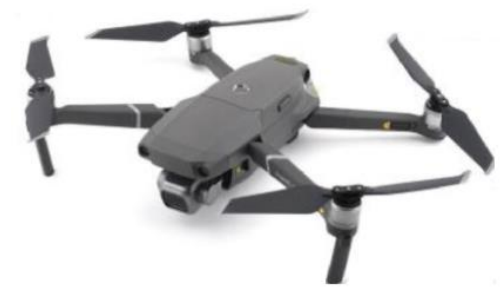

(a)

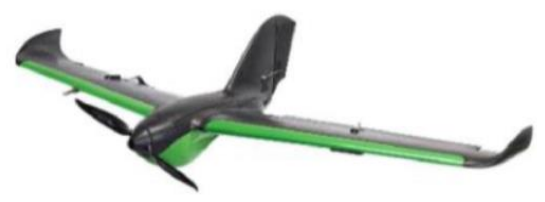

(b)

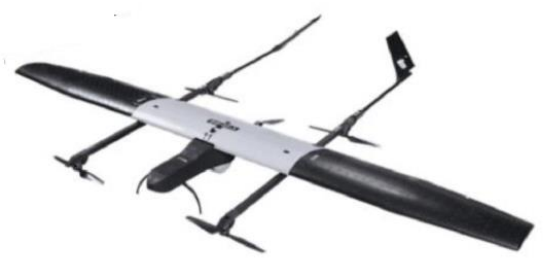

(c)

Figure 2. Examples of several types of UAV: (a) rotary-wing drone, (b) fixed-wing drone, (c) fixedwind hybrid vertical take-off and landing (VTOL) drone (modified from [19]).

Typically, UAVs present unique characteristics, being dynamic, agile, easy to repair, easy to deploy, and offering the potential to provide various measurements wherever and whenever required. They provide a low-cost alternative for collecting and providing information to intelligent systems that can undertake the necessary data analysis (e.g., 
real-time image or video analysis). UAVs will substantially promote the progress of farming, defense, insurance inspection, law enforcement, emergency assistance, pollution monitoring, disaster recovery, and package delivery.

The data are collected remotely through controllable devices installed on UAVs, each triggered via the platform. The collected data are processed locally onboard the UAVs or are offloaded to cloud servers, based on the required power. Deploying a practical UAVbased IoT framework requires an orchestrator platform that recognizes various contextual information about UAVs, such as flight trajectories, IoT equipment, and battery status.

Two main UAV communication networks exist: ad hoc UAV networks and UAVassisted aided communication networks. The former is characterized by a flexible topology and self-organized architecture, while the latter's main feature comprises flexible scheduling at the edge side of the network.

UAVs can collect data massively by being equipped with sensors of different sizes, forms, features, and capabilities. Many remote sensing devices for airborne and satellite platforms are now adopting miniature and low-cost versions for UAV platforms, such as multispectral, hyperspectral, short/medium-wave range cameras (e.g., thermal imaging camera), and light LiDAR. Table 1 gives a list of the sensors and cameras integrated with UAVs and their usefulness for monitoring various applications. The characteristics and specifications of each type differ according to specifications. For example, the RGB camera can be helpful for visual analysis, mapping and classifying objects and tracking animals and people. Moreover, UAVs with lightweight thermal infra-red sensors can deal with complex natural tracking such as monitoring volcanoes and forest fires, tracking animals, and in hydrothermal scientific studies.

Table 1. Examples of UAV onboard sensors and cameras.

\begin{tabular}{|c|c|c|}
\hline Sensor/Camera & Utility & Ref. \\
\hline RGB Camera & $\begin{array}{ll}\text { - } & \text { Visual analysis } \\
\text { - } & \text { Mapping } \\
\text { - } & \text { Object classification } \\
\text { - } & \text { Tehicle detection } \\
& \text { Tracking animals and people }\end{array}$ & [20] \\
\hline UAV LiDAR & $\begin{array}{l}\text { - } \quad \text { Vegetation canopy analysis } \\
\text { - } \quad \text { Estimation of forest carbon } \\
\text { - } \quad \text { Mapping cultural heritage } \\
\text { - } \quad \text { Building information }\end{array}$ & [21] \\
\hline Hyperspectral sensors & $\begin{array}{ll}\text { - } & \text { Land cover/land use mapping } \\
\text { - } & \text { Vegetation indices estimation } \\
\text { - } & \text { Biophysical, physiological, or biochemical parameter estimation } \\
\text { - } & \text { Detection, ind vegetadion disease } \\
\text { - } & \text { Assessment }\end{array}$ & [22] \\
\hline Lightweight cameras & $\begin{array}{l}\text { - } \quad \text { Visual analysis, vegetation detection and analysis, crop monitoring } \\
\text { - } \\
\text { - } \\
\text { - } \\
\text { Dinil moisture estion, water level measurement } \\
\text { Land cover/land use mapping }\end{array}$ & [23] \\
\hline Lightweight thermal infra-red sensors & $\begin{array}{l}\text { - Tracking creatures } \\
\text { - } \quad \text { Dolcanoes } \\
\text { - Hydrothermal studies, urban heat monitoring } \\
\text { - Islands and hazardous area tracking }\end{array}$ & [24] \\
\hline
\end{tabular}




\subsection{UAV-Enabled Services}

UAV technology's appealing flexibility and mobility characteristics have drawn attention in terms of integration with other networks as a way to address various challenging applications. UAVs can be a part of IoT networks, referred to as the Internet of Flying Things (IoFT) [25]. The IoFT can improve the availability of IoT services in areas with poor communication coverage and limited infrastructures. For instance, UAVs can provide FC and EC services and cellular communications in rural or developing regions and submarine environments. As a result, reaching hostile and congested environments has become easier and faster, thus improving emergent intervention wherever and whenever it is required. As support to communication networks, UAVs can be dispatched as peripheral nodes with various operating units, such as flying BSs, relay nodes, or end-node stations [7]. UAVs act as relay/terminal nodes related to disconnected nodes, stabilizing communication links in an ad hoc manner. A set of deployed UAVs can thereby form a flying ad hoc network (FANET), which is a subclass of the mobile ad hoc network (MANET) [26].

In IoT networks, UAVs can potentially adopt many recent cellular and non-cellular wireless communication networks to control the UAV on the one hand, and to enhance the coverage and latency services on the other. The most prominent communication protocols that can be used with UAVs are IEEE 802.11 (Wi-Fi), IEEE 802.16 (WiMax), IEEE 802.15.4 (LRWPAN), cellular networks (3G/4G), IEEE 802.15.1 (Bluetooth), LoRaWAN (LoRa), SigFox and narrowband IoT (NB-IoT) [27-31]. Typically, the choice of communication protocol depends on the desired achievable throughput, power consumption, range, implementation cost, reliability, delay, and security. Grouped according to application requirements, the characteristics of the most-used communication protocols are presented in [32]. As described in Table 2, there are various available communication protocols that can be used in UAV-enabled MEC. Each protocol is given a list of advantages and disadvantages regarding data rate, coverage, deployment cost, energy consumption, latency, spectrum availability, and the number of supported users. Thus, the use of communication technology depends literally on the application requirements.

Along with high mobility and scalability, UAVs can offer many potential opportunities in terms of enabling services such as pervasive connectivity, aerial intelligence, self-maintenance capabilities for communications, and sensor deployment. Therefore, UAVs act as airborne BSs [33], data collectors, relay nodes [34], jammers [35], monitors [36], edge- and cloud computing servers [37], and power suppliers [38] to support IoT applications. These will extend network coverage and provide diversified and flexible intelligence facilities for new potentialities in modern IoT applications. In summary, as illustrated in Figure 3, UAVs can facilitate a wide range of solutions in IoT applications. They can provide intelligent communications by establishing wireless networks in inaccessible and hazardous environments; they also enable parallel intelligent areal services, such as CC, EC, and LC. 
Table 2. Characteristics of the most-used communication technologies employed in UAV applications.

\begin{tabular}{|c|c|c|c|c|c|c|c|c|}
\hline Protocol & Max Data Rate & Max Range & Deployment Cost & Energy & Latency & Spectrum & Max Connectivity & Pros/Cons \\
\hline NB-IoT & $\begin{array}{l}\text { UL: } 158.5 \mathrm{kbps} \\
\text { DL: } 106 \mathrm{kbps}\end{array}$ & $15 \mathrm{~km}$ & $\begin{array}{l}\text { High } \\
(>15,000 \text { \$/BS) }\end{array}$ & $\begin{array}{l}\text { Low } \\
(3 \mu \mathrm{A} \text { rest, } \\
\text { Tx: } 74-220 \mathrm{~mA} \text {, } \\
\text { Rx: } 46 \mathrm{~mA})\end{array}$ & $1.6 \mathrm{~s}$ & Licensed Sub-GHz & $\begin{array}{l}\text { Massive } \\
\text { (>50,000 sensor } \\
\text { devices) }\end{array}$ & $\begin{array}{ll}+ & \text { Support remote healthcare monitoring, } \\
+ & \text { Energy-efficiency } \\
+ & \text { Provide fast, reliable network } \\
- & \text { High maintenance costs } \\
- & \text { Operational cost since it is on the } \\
& \text { licensed spectrum } \\
- & \text { Cost of SiM card purchase } \\
- & \text { No mobility support } \\
\end{array}$ \\
\hline LoRa & $\begin{array}{l}\text { LoRa CSS: } 0.3-5 \mathrm{kbps} \\
\text { FSK: } 50 \text { kbps }\end{array}$ & $\begin{array}{l}5 \mathrm{~km} \\
\text { (Urban) } 15 \mathrm{~km} \text { (Rural) }\end{array}$ & $\begin{array}{l}\text { High } \\
(100-1000 \text { \$/BS })\end{array}$ & $\begin{array}{l}\text { Very Low } \\
\text { (2 HA resting, } 12 \mathrm{~mA} \\
\text { Listening) }\end{array}$ & $>1 \mathrm{~s}$ & Sub-GHz ISM band & $\begin{array}{l}\text { Massive } \\
\text { (40,000 sensor devices) }\end{array}$ & $\begin{array}{ll}+ & \text { Long communication range } \\
+ & \text { Energy efficiency } \\
+ & \text { Low operational cost } \\
- & \text { Low data reliability } \\
- & \text { Interference issue } \\
- & \text { High deployment cost } \\
\end{array}$ \\
\hline SigFox & $\begin{array}{l}\text { UL: } 100 / 600 \text { bps } \\
\text { DL: } 600 \text { bps }\end{array}$ & $\begin{array}{l}10 \mathrm{~km} \text { (urban), } 50 \mathrm{~km} \\
\text { (rural) }\end{array}$ & $\begin{array}{l}\text { High } \\
(>4000 \text { \$ } / \mathrm{BS})\end{array}$ & $\begin{array}{l}\text { Very Low } \\
10-100 \mathrm{~mW} \\
(<0.01 \mathrm{~mA} \text { resting, Tx: } \\
28 \mathrm{~mA}, \mathrm{Rx}: 10.5 \mathrm{~mA})\end{array}$ & $10 \mathrm{~s}$ & Sub-GHz ISM band & $\begin{array}{l}\text { Massive } \\
\text { (40,000 sensor devices) }\end{array}$ & $\begin{array}{ll}+ & \text { Long communication range } \\
+ & \text { Energy efficiency } \\
+ & \text { Low operational cost } \\
- & \text { Interference issue } \\
- & \text { High deployment cost } \\
- & \text { Low data reliability } \\
- & \text { No mobility supports } \\
- & \text { Limited number of messages per day } \\
& (140 \text { Message/day, } 12 \text { bytes/message) }\end{array}$ \\
\hline ZigBee & $250 \mathrm{Kbps}$ & $<1 \mathrm{~km}$ & $\begin{array}{l}\text { Low } \\
(1-5 \$)\end{array}$ & $\begin{array}{l}\text { High } \\
30 \mathrm{~mA} \\
(3.16 \mu \mathrm{W}-1 \mathrm{~mW}) \\
-25 \text { to } 0 \mathrm{dBm}\end{array}$ & $15 \mathrm{~ms}$ & ISM & $\begin{array}{l}\text { Moderate } \\
\text { (255) }\end{array}$ & $\begin{array}{ll}+ & \text { Free license } \\
+ & \text { The node supports } \\
+ & \text { Flexible architecture and ease to install } \\
+ & \text { Support mesh networking } \\
+ & \text { Easy to control and monitor } \\
- & \text { Security issues } \\
- & \text { Energy consumption } \\
- & \text { Short transmission distance } \\
- & \begin{array}{l}\text { Prone to network interference due to the } \\
\text { use of } 2.4 \mathrm{GHz} \text { band }\end{array} \\
\end{array}$ \\
\hline $\begin{array}{l}\text { Bluetooth } \\
\text { IEEE 802.15.1 }\end{array}$ & $3 \mathrm{Mbps}$ & $100 \mathrm{~m}$ & $\begin{array}{l}\text { Low } \\
(5-10 \$)\end{array}$ & $\begin{array}{l}\text { Low } \\
(1 \mathrm{~W}) \\
1-10 \mathrm{~mW} \\
0-10 \mathrm{dBm}\end{array}$ & $3 \mathrm{~ms}$ & $\begin{array}{l}\text { ISM } \\
(2.4-2.4835 \mathrm{GHz})\end{array}$ & $\begin{array}{l}\text { Low } \\
\text { (Over } 1000 \text { in Bluetooth } \\
\text { mesh networking) }\end{array}$ & $\begin{array}{ll}+ & \text { Free of charge } \\
+ & \text { Simplicity } \\
- & \text { Low connectivity } \\
- & \text { Energy consumption } \\
- & \text { Short coverage } \\
- & \text { Security } \\
\end{array}$ \\
\hline BLE & 2 Mbps & $240 \mathrm{~m}$ & $\begin{array}{l}\text { High } \\
(>25 \$)\end{array}$ & $\begin{array}{l}\text { Low } \\
(0.01-0.5 \mathrm{~W})\end{array}$ & $50 \mathrm{~ms}$ & $\begin{array}{l}\text { ISM } \\
(2.4-2.5 \mathrm{GHz})\end{array}$ & Low & $\begin{array}{ll}+ & \text { Low power consumption } \\
+ & \text { Fast Connection establishment } \\
+ & \text { Free of charge } \\
+ & \text { Reliable for small size data transfer } \\
& \text { especially in IoT } \\
- & \text { Open to interception and attack } \\
- & \text { Short coverage } \\
- & \text { Limited data rates }\end{array}$ \\
\hline
\end{tabular}


Table 2. Cont.

\begin{tabular}{|c|c|c|c|c|c|c|c|c|}
\hline Protocol & Max Data Rate & Max Range & Deployment Cost & Energy & Latency & Spectrum & Max Connectivity & Pros/Cons \\
\hline $5 \mathrm{G} / 6 \mathrm{G}$ & $\begin{array}{l}1 \text { Gbps (5G) } \\
>10 \text { Gbps (6G) }\end{array}$ & $200 \mathrm{~m}$ & $\begin{array}{l}\text { High } \\
(>13,000 \$)\end{array}$ & Medium & $\begin{array}{l}1 \mathrm{~ms}(5 \mathrm{G}) \\
0.1 \mathrm{~ms}(6 \mathrm{G})\end{array}$ & Licensed cellular & $\begin{array}{l}\text { Massive } \\
1 \text { million } / \mathrm{km}^{2}(5 \mathrm{G}) \\
10 \mathrm{million} / \mathrm{km}^{2}(6 \mathrm{G})\end{array}$ & $\begin{array}{ll}+ & \text { Increased connectivity } \\
+ & \text { Lower latency } \\
+ & \text { Energy efficiency plans } \\
+ & \text { AI adoption } \\
- & \text { High deployment cost } \\
- & \text { Security and privacy issues } \\
- & \text { Limited coverage } \\
\end{array}$ \\
\hline $\begin{array}{l}\text { Wi-Fi } \\
\text { IEEE } 802.11\end{array}$ & $\begin{array}{l}\text { 11/54/300 Mbps } \\
7 \text { Gbps }\end{array}$ & $\begin{array}{l}250 \mathrm{~m} \\
100 \mathrm{~m}\end{array}$ & $\begin{array}{l}\text { High } \\
(100-1000 \text { \$ per BS) }\end{array}$ & Medium & $>20 \mathrm{~ms}$ & $\begin{array}{l}\text { Unlicensed ISM } \\
(2.4-5 \mathrm{GHz})\end{array}$ & $\begin{array}{l}\text { Moderate } \\
\text { (255) }\end{array}$ & $\begin{array}{ll}+ & \text { Mobility } \\
+ & \text { Cost } \\
+ & \text { Convenience } \\
- & \text { Range } \\
- & \text { Security } \\
- & \text { Reliability } \\
\end{array}$ \\
\hline $\begin{array}{l}\text { LTE M (Rel13 } \\
\text { and Rel14) }\end{array}$ & $\begin{array}{l}1 \text { Mbps } \\
\text { (LTE M Rel13) } \\
4 \text { Mbps } \\
\text { (LTE M Rel14) }\end{array}$ & $12 \mathrm{~km}$ & $\begin{array}{l}\text { High } \\
(>5000 \$)\end{array}$ & Medium & $>150 \mathrm{~ms}$ & $\begin{array}{l}\text { Licensed LTE frequency } \\
\text { band }\end{array}$ & $\begin{array}{l}\text { Massive } \\
\text { (20,000 sensor devices) }\end{array}$ & $\begin{array}{ll}+ & \text { Support remote healthcare monitoring } \\
+ & \text { Energy efficiency } \\
+ & \text { Provide fast reliable network } \\
- & \text { Limited network capacity }\end{array}$ \\
\hline
\end{tabular}




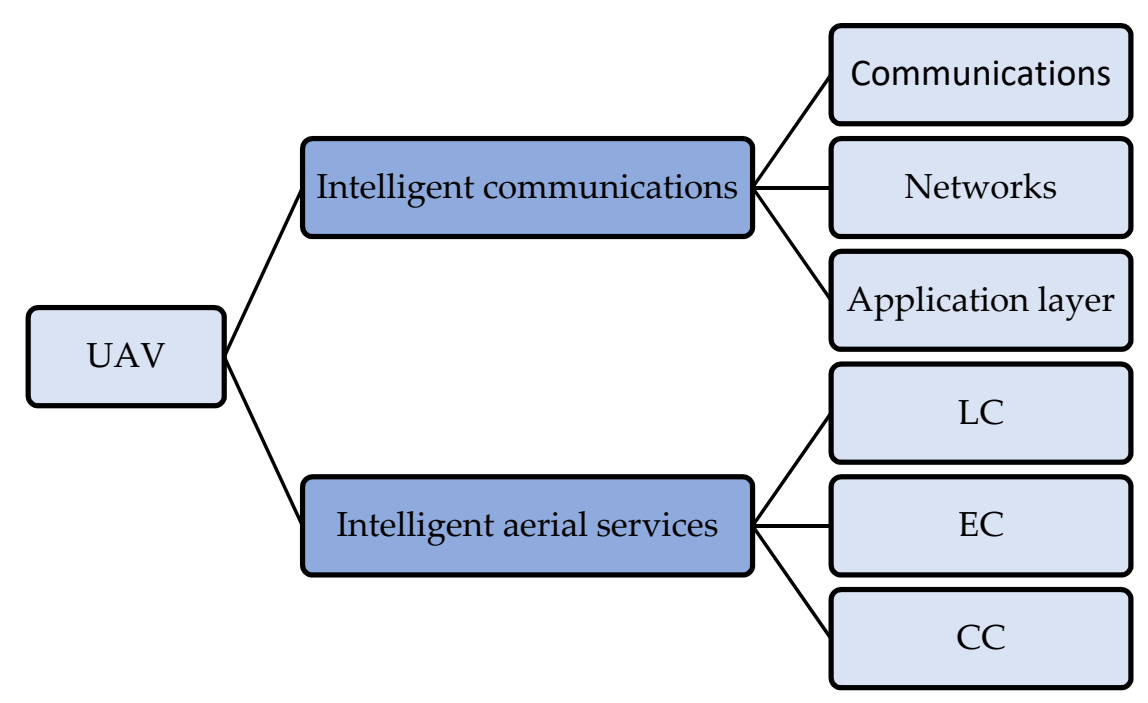

Figure 3. UAV-enabled intelligent services in IoT.

\subsection{UAV Applications}

Despite the strict restrictions on the civilian usage of drones, they play a promotional role in providing many facilities and enhancements in different application scenarios. Moreover, with the rapid development and emergence of new communication paradigms to achieve global connectivity, drones will positively facilitate many intelligent features. Table 3 shows a list of UAV applications in various domains, their advantages and disadvantages, and current research challenges.

- Agriculture:

Drones have been used to monitor large agricultural fields to detect specific diseases that threaten the quality of plants at an early stage. The implemented system can predict farm diseases in real-time by exploiting image processing and AI classification based on IoT architecture, using real-time data acquisition [39]. In ref. [40], the authors have proposed an architecture combining UAVs and wireless sensor networks (WSNs) with IoT approaches to provide precise ecological agriculture. Another architecture for crop mapping monitoring and disease assessment-based satellite imagery using the DL approach has been proposed to analyze the images acquired through UAV airborne multispectral sensors [41]. Additionally, UAVs can efficiently enhance the productivity of farming activities, as referenced in [42-44]. In addition, UAVs play an exciting role in crop monitoring by facilitating many tasks previously restricted to the laborer and farmer [45-47].

- Industry 4.0:

The increasing demand for fast and low-cost productivity has encouraged the industrial and research communities to incorporate robotics into the industrial process. UAVs have recently attracted the attention of industrial solution providers in response to the increased need for productivity; for instance, the authors proposed an industrial IoT-based system that collects data by a UAV camera and sends it to a cloud server for processing [48]. The design aims to monitor the industrial zone to prevent undesirable incidents in the production process, relying on the robust intelligence of computer vision (CV) approaches $[49,50]$. The mining industry has also increased the level of interest in drones for 3D mapping, mine safety, and mine management [51].

- Environment:

UAVs are being adopted as a means to monitor and survey various environmental areas. They can be used for the monitoring and data aggregation of archeological sites. For example, the authors of [52] have reported that UAVs can present accurate and precise images of the studied archeological space, compared to terrestrial measurements. UAV 
technologies can also be deployed in ecological monitoring systems to track wildlife in heterogeneous territories and topographically challenging areas as a supporting platform for traditional techniques [53]. Besides, this technology may facilitate geological researchers when collecting remote data about many species and animals [54]. Moreover, the authors of [55] proposed an intelligent model based on UAV, dedicated to monitoring several types of plants using the latest computer vision algorithms and cloud computing technologies. UAVs are also used to track wild animals over large areas [56].

- Health and emergency:

Drones can offer several benefits for public health emergencies, for instance, tackling epidemics and contagious diseases such as COVID-19. For instance, during the COVID-19 pandemic, some governments and countries adopted drones to survey the rapid spread of the virus, diagnose patients, and then predict the mortality risk factor by applying AI approaches to the collected data. The drones have been used to observe crowds, broadcast critical public announcements and information, spray disinfectants, and carry out extensive temperature measurements in several residential areas [57]. Additionally, the lifeguard community uses UAVs for search and rescue missions requiring rapid intervention. Their versatility also allows obtaining a quick overview of the rescue situation instead of using human-crewed airplanes that may take extra time to deploy [58]. Relying on UAV-enabled MEC, AI, and CV schemes could lead to new applications that simulate visual reality, thus enabling faster and real-time decisions in emergency interventions. For instance, UAVs typically detect obstacles by flying over them, gathering data, extracting the most prominent features, and making decisions based on machine vision and pattern recognition technologies. In this manner, people with visual impairments can maneuver comfortably. This technology is expected to be a key solution in the healthcare revolution [58].

- Smart cities and smart homes:

The urban environment is expected to acquire a massive number of dynamically connected devices due to diverse activities wherein UAVs as mobile vehicles will have many pivotal roles, including real-time traffic control [59], transportation [60], infrastructure control and management [61], and buildings observation [62]. Accordingly, UAVs are expected to provide communication services to heterogeneous smart devices in urban areas, to improve the smart city from application perspectives [63]. For example, the authors of [64] have proposed an architecture for 5G hierarchical IoT networks where the UAVs play a fundamental role as a data fusion center, formation controller, and network gateway. In another context, UAVs were used to perform lightweight parts delivery to workstation operators within a manufacturing plant, where GPS could not provide a practical solution for indoor positioning, in addition to intelligent shipping, intelligent monitoring, and intelligent control [65].

- Natural disaster tracking:

During natural disasters, a rapid and effective response is required to assist the population, avoid increasing the number of victims, and minimize the economic impact. UAVs can respond quickly to earthquake help requests, find missing people, and facilitate the monitoring and rescue of victims in danger of drowning during floods [66,67]. Collecting a large amount of data from different sensors deployed in the environment, using UAVenabled MEC with AI algorithms, can help to rapidly predict and intervene in natural disasters such as tornadoes, volcanic eruptions, tsunamis, and storms.

- Construction:

UAV-enabled MEC and computer vision techniques are promising solutions for the condition assessment of civilian and public infrastructures. UAVs are being used increasingly in many construction tasks, such as building inspections [68], building condition monitoring, damage assessments of buildings after disasters [69], public site surveying and mapping [70], safety inspections of construction sites and workers, and monitoring the progress of construction [71]. 
Table 3. Comparative study of UAV application objectives.

\begin{tabular}{|c|c|c|c|c|}
\hline Application Domains & Objectives & Pros/Cons & Future Insights & Ref. \\
\hline Agriculture/Precision agriculture & $\begin{array}{ll}\checkmark & \text { Optimizing spraying process } \\
\checkmark & \text { Crop monitoring } \\
\checkmark & \text { Crop maturity monitoring } \\
\checkmark & \text { Detection and prediction of various } \\
& \text { crop diseases } \\
\checkmark & \text { Irrigation management } \\
\checkmark & \text { Artificial pollination } \\
\checkmark & \text { Greenhouse temperature and humidity } \\
& \text { monitoring } \\
\checkmark & \text { Water assessment }\end{array}$ & $\begin{array}{ll}+ & \begin{array}{l}\text { Basic knowledge and skills are required to } \\
\text { operate }\end{array} \\
+ & \text { UAVs with greater capabilities cost more } \\
- & \text { Weather conditions limit the utilization } \\
\text { of UAVs } & \\
- & \text { Limited energy of drones }\end{array}$ & $\begin{array}{ll}\checkmark & \text { Multi-UAV integration } \\
\checkmark & \text { Difficult to fly in harsh conditions } \\
\checkmark & \text { Energy efficiency } \\
\checkmark & \text { UAV-based autonomous decisions } \\
\checkmark & \text { Autonomous decision-making based on DL } \\
\checkmark & \text { Short-range coverage of BLE-enabled UAVS } \\
& (100 \text { m) } \\
\checkmark & \begin{array}{l}\text { Interference (Wi-Fi, Zigbee, and regular } \\
\text { Bluetooth technologies) }\end{array} \\
\checkmark & \begin{array}{l}\text { Interoperability with new raised } \\
\text { communication technologies }\end{array} \\
\checkmark & \begin{array}{l}\text { More work on using AI to model crop growth } \\
\text { and disease management is expected based on } \\
\text { on-farm and meteorological data }\end{array} \\
\checkmark & \begin{array}{l}\text { When developing laws and regulations for IoT } \\
\text { in agriculture, the involvement of the }\end{array} \\
\text { government or agricultural department must } \\
\text { be assured }\end{array}$ & [72] \\
\hline Environment/Natural disasters & $\begin{array}{ll}\checkmark & \text { Monitoring, forecasting, early warning systems } \\
\checkmark & \text { Disaster information fusion } \\
\checkmark & \text { Search and rescue missions } \\
\checkmark & \text { Damage assessment } \\
\checkmark & \text { Standalone communication systems }\end{array}$ & $\begin{array}{ll}+ & \text { Reducing cost and economic losses } \\
+ & \text { Fast intervention } \\
+ & \text { Easy to install } \\
- & \text { Bad weather conditions } \\
- & \text { Limited battery } \\
- & \text { Limited flying time }\end{array}$ & $\begin{array}{l}\text { Data fusion and handover issues } \\
\text { Improve the UAV localization systems } \\
\text { Design efficient schemes for UAVs }\end{array}$ & [32] \\
\hline
\end{tabular}


Table 3. Cont

$+\quad$ Enhance energy efficiency of IoT devices on the ground in intelligent cities

Delivering collected data in real-time

Enhancing the quality of life

Reducing cost and economic losses

Low latency services (fog-computing architecture)

- Drone equips many different IoT devices,

which affect processing services and energy
Big data processing issues

$\checkmark \quad$ Ethical and privacy

$\checkmark \quad$ Licensing and legislation

Development of automated image dat compression algorithms,

Development of a network-centric infrastructure Precision flying should be improved under al weather conditions

Communication constraints

Security threats should be addressed by using new emerged technologies (i.e., blockchains) Applying ML techniques between edge/fog computing (drone) and the IoT cloud

$\checkmark$ Real-time processing of swarms of drones' photos, recorded movies, and data collection necessitates implementing and designing efficient power-distributed algorithms

Air traffic control

$\checkmark \quad$ Bad weather conditions

$\checkmark \quad$ Liability insurance

$\checkmark \quad$ Legislations

Logistics and Transportation

$\checkmark \quad$ Food delivery services

Faster delivery of food

Data security on the transmission line
Navigation system should be developed to

enable autonomous flying of UAVs

Delivery of lightweight commercial product
- Drones' batteries are limited are required

on-demand communication

Possibility of coverage and network

capacity enhancement

IoT support

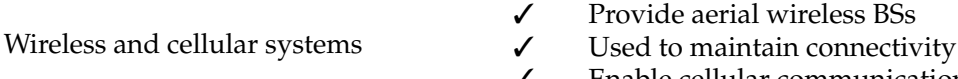

Enhancing the performance of delivering

communication services

$+\quad$ Maintaining QoS

Battery limitation

Interference

Interoperability 
- Wireless and cellular networks:

Many efforts have been dedicated to harnessing the full potential of UAV communications for cellular and wireless communications in the networks industry. The research community explored the opportunity of UAV-mounted utilization via flying relays and BSs that can dynamically reposition themselves to boost network coverage, increase spectral efficiency, and improve user QoS. The UAVs enable low-altitude supports beyond line-ofsight (LOS) control and reliable communications [72]. Furthermore, UAVs can bring many facilities to cellular IoT-based networks by providing processing facilities near the ground for users.

\subsection{UAV-Enabled and-Assisted MEC Architecture}

Centralized networks are easy to maintain because they have just one point of failure. However, due to the instability of the system categories, this design is insufficient to respond to ubiquitous applications. A single issue affecting the core server might destroy the entire system. Unlike centralized networks, distributed networks are deemed more stable due to their decentralized architecture, since the entirety of the information system is dispersed among a vast number of nodes that maintain similar conditions. This feature improves distributed system security by making it challenging to target many users.

Furthermore, blockchain is a peer-to-peer (P2P) distributed ledger that provides a secure, immutable, transparent, and trustless environment due to its distributed architecture. Integrating this approach with UAVs allows them to record their data in the chain of blocks as a transaction, ensuring their trustworthiness [74]. The essential foundations of blockchain technology are intelligent contracts and consensus mechanisms. This method eliminates the requirement for a trusted third-party system to protect data privacy since consensus techniques maintain data integrity. The use of cryptographic methods guarantees that data on blockchains are secure. These exciting blockchain characteristics increase the applicability of UAVs in a variety of civil and mission-critical applications that use UAV-enabled MEC architecture. Moreover, distributed systems outperform centralized systems in terms of network speed, since the information is not stored in a central location, and the number of users attempting to access a server is more significant than the server can support, causing long waiting times and slowing down the system for decentralized architecture.

Moving all computing activities to the cloud has proven an effective mechanism to process data since the cloud offers greater processing capacity than network edge devices. However, data-processing speeds have risen rapidly due to the size of communicated data and the networks' bandwidth. Therefore, CC suffers from bottlenecks due to the massive volume of data generated in real time. Cameras in UAVs capture a tremendous amount of video data, which the system should process in real-time to enable good decisionmaking. Sending data from UAVs to the cloud server would mostly result in longer delays. Deploying a large number of IoT devices, such as UAVs, would restrict bandwidth, reliability and security. Therefore, processing data at the network edge would yield shorter response times, more efficient processing, and less pressure on the network.

MEC is widely acknowledged as a vital technology to implement many methods for next-generation IoT applications. Due to their versatility and ease of deployment, UAVs can play various roles in delivering edge computing services. UAV-enabled MEC designs can be classified and dedicated to a specific application scenario. A UAV can be considered as a mobile EC server, an IoT node, or a relay. Firstly, UAVs can participate in the system as mobile devices when they offload their computing to an MEC server. Secondly, the UAV can act as the MEC responsible for monitoring a group of mobile end nodes. Thirdly, the UAV can serve as a relay or gateway between mobile end nodes and an MEC server.

The UAV may be regarded as a specific user that could offload those computing tasks exceeding its memory and processing capacity to a MEC server, as shown in Figure 4a. UAVs typically have limited battery capacity and insufficient memory and computing power to execute complex computational tasks. As a result, offloading their processing to 
the ground MEC server for computation may increase its battery life. In another situation, as shown in Figure $4 b$, the UAV may keep the MEC server on board and fly to assist the ground-based users with the task of computing once the ground-based users offload their computation responsibilities to the latter. In the third scenario depicted in Figure 4c, the UAV serves as a central relay, assisting mobile users to offload their computing tasks to a specific MEC server.

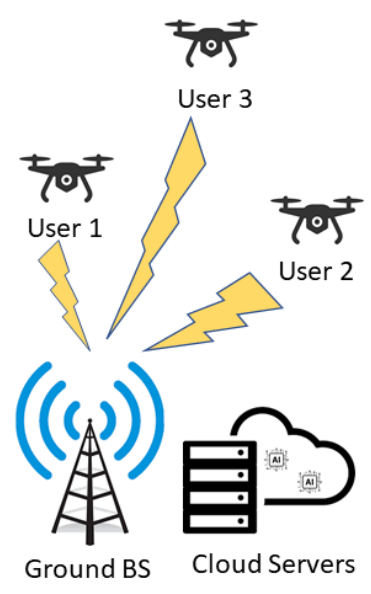

a) The scenario UAVs have computation task.
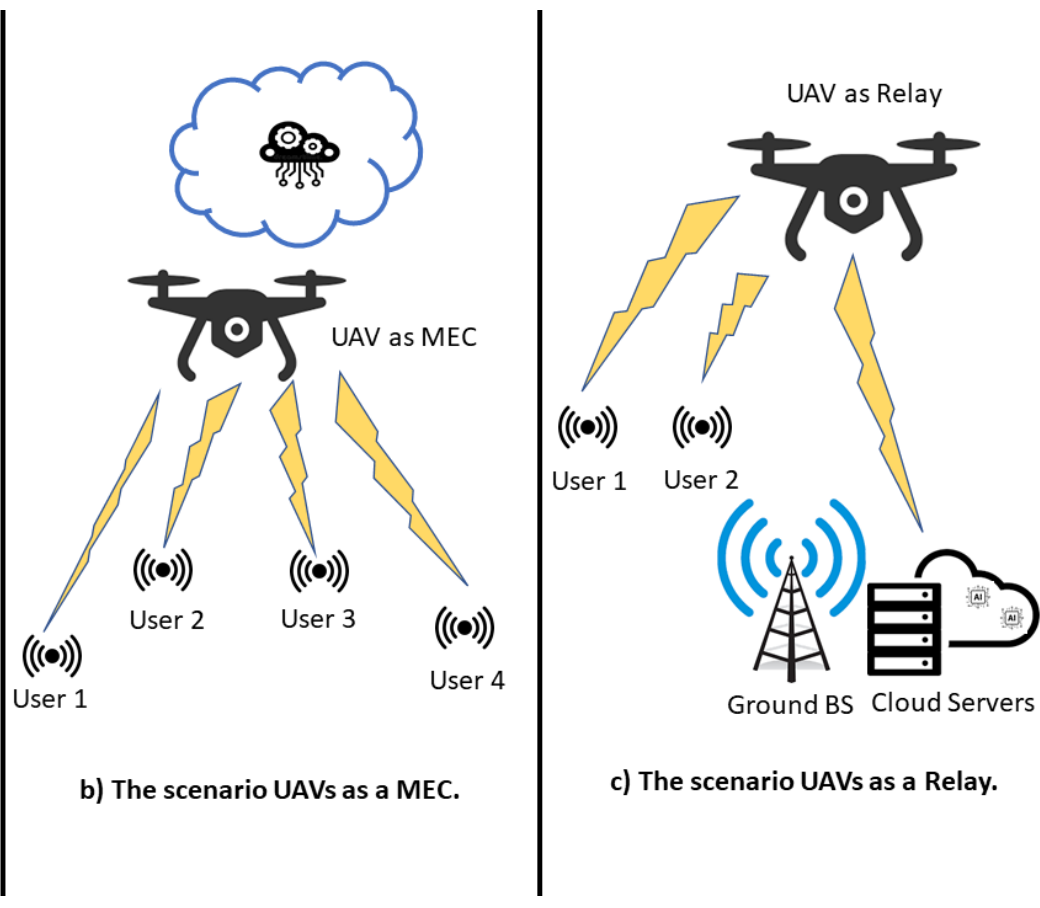

Figure 4. UAV-enabled architecture.

Despite the substantial benefits afforded by the architecture of UAV-enabled MECs to provide low latency and high-reliability services to IoT devices, numerous challenges remain. These challenges are found in intra-UAV communication, UAV security, air data security, data storage, and management. Moreover, the coordination between UAVs and ground-based users requires considerable improvement due to the system's mobility characteristics, which complicate communication. Another significant limitation of UAVs is found in their low onboard battery capacity. In addition to the energy spent in hovering, accelerating/decelerating, and climbing up/down, additional energy is consumed due to onboard computations. Therefore, designing efficient methods for resource and energy management is urgently required.

Furthermore, one of the major design problems regarding computation task requirements is the joint trajectory design in UAV-enabled MEC networks. In this regard, research efforts should emphasize how to forecast mobile user movement and follow the trajectory so that computing activities can be offloaded quickly, and computation results may be sent back to users on time. The trajectory design is especially challenging when dealing with multi-UAV-enabled MEC services. Another challenge is found in the integration of the blockchain into UAVs. As standalone technologies, UAVs face many challenges such as privacy issues, air traffic violations, quantum attacks, $\mathrm{ML}$, and algorithmic game-theory-based attacks [13].

\section{UAV-Enabled and Assisted MEC State of the Art}

The rapid spread of smart devices has led to intensive computation in many applications, such as virtual reality, face and object recognition, and automatic driving. This poses a severe challenge when intelligent devices with limited computing and energy 
resources need to perform the required tasks locally [75]. CC can be considered as an expensive solution to overcome these impairments. In addition, the CC solution suffers from high communication expenses and high transmission delays due to the core network's involvement. To this end, some prominent alternatives have been proposed to explore the latency delay, including recalling the computation resources from the cloud to the edge of the network.

As an extension of the edge concept, recently, MEC was intensively investigated. MEC can bring information technology (IT) services and cloud-computing capabilities to a mobile network, guaranteeing ultra-low delays and offering high bandwidth to the user [76]. UAVs can be embedded with lightweight CPUs (central processing units) or GPUs (graphics processing units) to offer computation services. Therefore, UAVs fly MEC servers in the sky, offering controllable mobility and high-quality communication links, supplying the existing MEC servers on the ground. In this regard, we summarize the state-of-the-art situation regarding UAVs' possibilities as MEC enablers or as part of a MEC system in an IoT environment where end-nodes and actuators cannot deal with the generated data onboard.

The research on UAV-enabled MEC or UAV-assisted MEC is in its early phases, including using the fog and edge computing concepts instead of cloud computing to enhance latency and computation performances in IoT users. A summary of related research activities regarding UAV opportunities as an MEC enabler or as part of a MEC system in the IoT is listed and highlighted in Table 4.

Table 4. UAV-enabled MEC and assisted MEC research activities.

\begin{tabular}{|c|c|c|c|}
\hline Ref. & Year & Evaluated Performance Metrics & Summary \\
\hline [77] & 2021 & $\begin{array}{l}\text { - Communication security issue } \\
\text { in the dual-UAV-MEC system }\end{array}$ & $\begin{array}{l}\text { A secure communication mechanism was created, dedicated to the } \\
\text { dual-UAV-MEC system. The main objective of this task was to } \\
\text { maximize the user's security and computing capacity by optimizing } \\
\text { the resources and trajectory of the UAV server. The authors relied on } \\
\text { mathematical techniques, including the sequential convex } \\
\text { approximation (SCA) and block coordinate descent (BCD) algorithms } \\
\text { to enhance UAV-enabled MEC security and computing capacity. }\end{array}$ \\
\hline [37] & 2020 & - Computation offloading & $\begin{array}{l}\text { A hybrid DL and fuzzy c-means clustering-based algorithms were } \\
\text { proposed to predict the positions of ground-based users and UAVs in } \\
\text { a hybrid MEC (H-MEC) network. As a result, IoT devices can } \\
\text { efficiently offload their intensive tasks to the UAV servers. }\end{array}$ \\
\hline [78] & 2020 & $\begin{array}{l}\text { - } \quad \text { Computation offloading } \\
\text { - } \quad \text { Latency }\end{array}$ & $\begin{array}{l}\text { The authors proposed an intelligent task-offloading algorithm (iTOA) } \\
\text { for UAV-enabled MEC services. The proposed approach intelligently } \\
\text { perceives the network's environment and decides the offloading } \\
\text { action using the deep Monte Carlo tree algorithm. This method } \\
\text { outperforms game theory and greedy search-based methods in } \\
\text { latency performance. }\end{array}$ \\
\hline [79] & 2021 & $\begin{array}{l}\text { - } \quad \text { Computation offloading } \\
\text { - } \quad \text { Latency }\end{array}$ & $\begin{array}{l}\text { UAVs were adopted in } 5 \mathrm{G} \text { mobile networks to reduce the end-to-end } \\
\text { latency and improve communication reliability. Using UAVs with } \\
\text { MEC has provided effective traffic management, resulting in } \\
\text { decreased latency and better offloading operations. }\end{array}$ \\
\hline [80] & 2021 & - $\quad$ Energy & $\begin{array}{l}\text { A novel system called GEESE was proposed to provide computation } \\
\text { services on the network's edge by integrating cloudlets on multiple } \\
\text { aerial UAVs. The system performance has been investigated to } \\
\text { understand the relationship between energy efficiency and } \\
\text { computation task offloading. }\end{array}$ \\
\hline
\end{tabular}


Table 4. Cont

\begin{tabular}{|c|c|c|}
\hline Ref. & Year & Evaluated Performance Metrics \\
\hline [81] & 2021 & $\begin{array}{ll}\text { - } & \text { Energy } \\
\text { - } & \text { Computation handling for } \\
\text { innovative logistic applications }\end{array}$ \\
\hline
\end{tabular}

Summary

The energy management of UAV-enabled MEC was investigated in the context of a realistic autonomous delivery network. A computational management solution was designed explicitly for MEC-based task offloading and scheduling strategies. The integrated solution includes both static task offloading and dynamic resource scheduling. The experimental results have revealed that the system can handle a greater UAV payload while using less energy.

The authors addressed the issue of the energy consumption of IoT devices in UAV-enabled MEC networks. The energy and offloading requirements have been improved by optimizing the UAV trajectory planning, communications, and computing resource allocation.

[82] $2021 \quad \bullet \quad$ Energy consumption

A UAV-assisted MEC system, in which the UAVs acts as edge servers,

$\begin{array}{lll}\text { [83] } 2020 & \bullet & \text { Energy consumption } \\ & \bullet & \text { Trajectory planning for UAVs }\end{array}$
was designed to provide computing services for IoT devices. The proposed approach is based on a k-means clustering algorithm to minimize the energy consumption of the system by planning the trajectories of UAVs efficiently.

The Lagrangian duality method and successive convex approximation techniques were proposed to reduce UAV-assisted MEC computational complexity. The proposed approach aimed to

[84] $2020 \quad$ - Energy consumption minimize total energy consumption, including communication-related energy, computation-related energy, and UAV energy. This was achieved by optimizing bits allocation, time-slot scheduling, power allocation, and UAV trajectory design.

The authors proposed a UAV-enabled MEC architecture in which the UAVs were considered as MEC servers. The objective of this approach was to minimize the energy consumption of both UAV and ground-based users by scheduling computation resources and optimizing the UAV trajectories.

The authors have investigated a UAV-enabled MEC system based on the time division multiple access (TDMA) model. A TDMA-based scheme was proposed to minimize the user's energy consumption by optimizing the UAV coordinates, time-slot allocation, and task partitioning.

A two-layer optimization method was provided to address deployment and task-planning issues in a UAV-enabled MEC system.

[87] 2020 - Energy consumption This approach proved efficient for power consumption optimization. The proposed method was based on a differential evolution algorithm with a removal agent.

A multi-UAV architecture was proposed in which the UAVs act as computer servers to process the ground-based user's data and to minimize energy consumption. In this approach, a two-layer strategy was used to optimize the UAV's task scheduling based on dynamic scheduling-based bidding, whereas the second layer addressed bits allocation and the UAV's flight path.

UAV-enabled MEC architecture based on the Markov decision process (MDP) was proposed to optimize mobile users' energy demands and task offloading. In this work, the UAVs were considered intelligent mobile users.

An algorithm based on block coordinate descent and successive convex approximation techniques was proposed to optimize data offloading. By considering a single UAV, the proposed system improved the tasks of offloading and energy consumption. 
Table 4. Cont.

\begin{tabular}{ccll}
\hline Ref. & Year & Evaluated Performance Metrics \\
\hline [91] & & & \\
& 2020 & $\bullet$ & Latency \\
& & & \\
& & & \\
& & $\bullet$ & Latency \\
{$[92]$} & 2020 & $\bullet$ & $\begin{array}{l}\text { Offloading tasks } \\
\end{array}$ \\
& & $\bullet$ & Security \\
& & $\bullet$ & $\begin{array}{l}\text { Latency } \\
\text { [93] }\end{array}$ \\
& 2018 & $\bullet$ & $\begin{array}{l}\text { Reliability } \\
\text { Computation }\end{array}$ \\
\hline
\end{tabular}

Summary

A UAV-assisted MEC method was proposed, in which the UAVs acted as intermediate devices between the ground-based users and MEC servers. This method leveraged airborne computing and storage facilities to minimize the execution time of offloaded tasks for IoT users. Therefore, the task scheduling and flight path of the UAVs were jointly optimized.

The authors proposed a low-complexity iterative algorithm to optimize security and privacy, subject to latency, offloading, and energy constraints. This method was proposed to optimize the UAV location, the user's transmission power, UAV jamming power, offloading ratio, UAV computing capacity, and offloading user association.

An air/ground framework for MEC was proposed to combine the capabilities of ground vehicles with UAVs in terms of communication, computing, and storage.

[11] 2020 - Limited offloading tasks

An automatic offloading approach based on the MEC architecture has been proposed to deal with the limited processing capabilities of MEC servers and ground-based users. The UAVs have been used to cache the generated data from the IoT devices and then send it to the MEC servers, which operate in a private blockchain network.

\begin{tabular}{|c|c|c|}
\hline [94] & 2017 & - $\quad$ Network connectivity \\
\hline [95] & 2019 & $\begin{array}{ll}\text { - } & \text { Offloading tasks } \\
\text { - } & \text { QoS }\end{array}$ \\
\hline [96] & 2021 & $\begin{array}{l}\text { - } \quad \text { Path planning } \\
\text { - } \quad \text { QoS }\end{array}$ \\
\hline
\end{tabular}

A UAV-based MEC infrastructure was proposed to improve the network connectivity in uncovered areas. The system helped terrestrial users to compute their tasks in circumstances such as natural catastrophes or in rural locations without communication coverage.

UAVs were used as MEC-aided systems in wireless communication systems to ensure high QoS for ground-based users. The UAVs flew around the users to provide computing services in an orthogonal way over time.

A multi-UAV-enabled MEC platform was investigated to assess RL QoS and path planning. The study studied the autonomy and self-hovering ability of a network of UAVs relying on RL algorithms.

Mobile peripheral computing was deemed a promising technique to address computationally intensive issues. UAV-assisted MEC based on NOMA (non-orthogonal multiple access) can provide flexible

- $\quad$ Power consumption

[97] 2019 - Computing services computing services for mobile terminals (MTs) in large-scale access networks, as NOMA methods can be adaptive to massive

- Resources allocation connectivity. In this work, an optimization approach was presented to minimize the power consumption of MTs by jointly optimizing trajectory, task offloading, computing, and resource allocations.

\begin{tabular}{|c|c|c|c|}
\hline [98] & 2018 & $\begin{array}{l}\text { - } \quad \text { Propagation losses } \\
\text { - } \quad \text { Energy harvesting }\end{array}$ & $\begin{array}{l}\text { The authors proposed a UAV-enabled MEC and wireless-powered } \\
\text { architecture to tackle propagation packet loss in the IoT era. }\end{array}$ \\
\hline [8] & 2019 & - $\quad$ QoS & $\begin{array}{l}\text { A joint architecture using the edge and cloud models based on UAV } \\
\text { swarms was proposed to assure high service qualities in } \\
\text { resource-intensive and real-time applications. }\end{array}$ \\
\hline [10] & 2018 & $\begin{array}{ll}- & \text { Radio access networks } \\
\text { - } & \text { Edge caching and computing }\end{array}$ & $\begin{array}{l}\text { An AGMEN (aerial-ground integrated mobile edge network) } \\
\text { architecture was proposed to address many EC network issues, such } \\
\text { as communication, computing, and caching. The objective of this } \\
\text { approach was to optimally allocate computing and storage resources. } \\
\text { The authors deployed a set of UAVs to ensure spatial and temporal } \\
\text { coverage, as well as ensuring data delivery for mobile IoT users. }\end{array}$ \\
\hline [99] & 2021 & $\begin{array}{ll}- & \text { Real-time } \\
- & \text { Computation overhead }\end{array}$ & $\begin{array}{l}\text { A traffic monitoring system based on the multi-EYE method was } \\
\text { presented to detect and estimate the velocity of unmanned vehicles using } \\
\text { aerial image tracking. The image processing was executed in real-time } \\
\text { on an embedded edge-computing platform installed on the UAV. }\end{array}$ \\
\hline
\end{tabular}


Table 4. Cont.

\begin{tabular}{|c|c|c|c|}
\hline Ref. & Year & Evaluated Performance Metrics & Summary \\
\hline [100] & 2020 & - $\quad$ Reduce cost & $\begin{array}{l}\text { The concept of EC with UAV was used to perform mapping and } \\
\text { lodging assessment in a rice crop without human interaction to } \\
\text { reduce maneuvering cost and improve the quality of productivity. } \\
\text { The process relies mainly on UAV as an edge server to execute the } \\
\text { DNN algorithm while processing the images. }\end{array}$ \\
\hline [101] & 2021 & - $\quad$ Resource allocation & $\begin{array}{l}\text { In this work, AI methods have been used in a UAV-enabled MEC } \\
\text { based on the NOMA system. This approach allows terrestrial mobile } \\
\text { users to offload their computing duties intelligently. This is intended } \\
\text { to increase connectivity and minimize transmission latency and } \\
\text { power consumption. }\end{array}$ \\
\hline [102] & 2019 & $\begin{array}{ll}\text { - } & \text { Resource allocation } \\
\text { - } & \text { Energy }\end{array}$ & $\begin{array}{l}\text { The authors intended to extend a } 5 G \text { network for a video surveillance } \\
\text { application using a flying ad hoc network consisting of UAVs and EC } \\
\text { services. The authors aimed to increase the performance of the entire } \\
\text { MEC aerial platform, reduce latency, and ameliorate the reliability of } \\
\text { the system's source usage. }\end{array}$ \\
\hline [103] & 2021 & $\begin{array}{l}\text { - } \quad \text { Resource allocation } \\
\text { Power control in a MEC } \\
\text { based system }\end{array}$ & $\begin{array}{l}\text { Two approaches were proposed to deal with resource allocation and } \\
\text { power control in a UAV-enabled MEC system. The first approach was } \\
\text { a centralized multi-agent RL (MARL) algorithm, which has been } \\
\text { used to optimize the system's power consumption and resource } \\
\text { allocation. The second approach is a federated multi-agent } \\
\text { reinforcement learning (MAFRL) algorithm, which has been } \\
\text { proposed to guarantee security and privacy. }\end{array}$ \\
\hline
\end{tabular}

\begin{tabular}{|c|c|c|c|}
\hline [104] & 2019 & - $\quad$ Security & $\begin{array}{l}\text { A cyber-defense approach based on a non-cooperative game } \\
\text { algorithm was proposed to protect a UAV-enabled MEC from } \\
\text { network and offloading attacks. }\end{array}$ \\
\hline [105] & 2019 & $\begin{array}{ll}\text { - } & \text { Security } \\
\text { - } & \text { Privacy }\end{array}$ & $\begin{array}{l}\text { A MEC server-based authentication framework was proposed to be } \\
\text { integrated into UAVs. This was mainly to enhance the privacy and } \\
\text { authentication of UAVs. }\end{array}$ \\
\hline [106] & 2020 & $\begin{array}{ll}\text { - } & \text { Task offloading } \\
\text { - } & \text { Resource allocation }\end{array}$ & $\begin{array}{l}\text { A UAV-assisted multi-user MEC system based on frequency division } \\
\text { multiple access (FDMA) under Rician's fading channels was proposed } \\
\text { to test task offloading and resource allocation performances. }\end{array}$ \\
\hline [107] & 2019 & - $\quad$ Task offloading & $\begin{array}{l}\text { A theoretical game strategy based on three types of players was } \\
\text { proposed to formulate and solve the problem of offloading task } \\
\text { calculations in UAV-enabled MEC networks. }\end{array}$ \\
\hline [108] & 2018 & $\begin{array}{ll}\text { - } & \text { Task offloading } \\
\text { - } & \text { Throughput }\end{array}$ & $\begin{array}{l}\text { In this work, a UAV was used as a mobile edge server to manage } \\
\text { offloading processing tasks in real-time for ground-based users. A } \\
\text { hybrid scheme based on a semi-Markov decision process and DL was } \\
\text { proposed to maximize the throughput requirement. }\end{array}$ \\
\hline [109] & 2020 & - $\quad$ Task offloading & $\begin{array}{l}\text { Two offloading schemes for multiple UAVs-enabled MEC networks } \\
\text { were proposed to optimize computation time and energy } \\
\text { consumption. A game theory model was adopted to validate the } \\
\text { proposed strategies. }\end{array}$ \\
\hline [110] & 2018 & $\begin{array}{ll}\text { - } & \text { Transmission power } \\
\text { - } & \text { UAV trajectory }\end{array}$ & $\begin{array}{l}\text { A UAV-assisted MEC environment over the social internet of vehicles } \\
\text { (SIoV) with a three-layer integrated architecture was adopted. Total } \\
\text { utility maximization was achieved by jointly optimizing the } \\
\text { transmission power of the vehicle and the UAV trajectory. }\end{array}$ \\
\hline
\end{tabular}

\section{UAV-Enabled MEC and Assisted MEC Based on AI}

MEC is an effective method for overcoming time-constrained computing challenges in resource-constrained mobile IoT devices. The objective is to bring CC services closer to customers to minimize latency and backhaul network congestion. UAVs with storage units, processing, and communication capabilities can function as flying MEC servers at the IoT system's edge. In this context, resource-constrained IoT devices may offload their 
computing activities, either partially or totally, to MEC-server-mounted UAVs through direct line-of-sight connectivity. Nevertheless, the system faces many challenges related to task prediction, UAV deployment, users' association, signal processing, and joint resource allocation. Considering the EC architecture, UAVs with flexibility and mobility are likely to deliver decentralized solution perspectives significantly. The flying edge architecture is intended to improve the CC capabilities of latency-sensitive and real-time IoT applications. This enables computing offloading at the edge of IoT devices rather than centralized data centers, reducing communication latency and facilitating real-time decision-making and control. Since the IoT system operates with many end-devices, a large quantity of data is offloaded to the edge devices; this requires developing mechanisms that manage and exploit the data for various purposes, such as auto-maintenance, self-monitoring, and prediction. There is a considerable difference between EC devices and centralized cloud servers regarding their processing capacities and memory, limiting multiple AI technologies for data processing at the edge. Therefore, numerous criteria should be considered when incorporating any AI technique to profit from their robustness, due to memory capacity and energy consumption constraints [111]. EC delivers resources in a decentralized manner, allowing faster addressing of user demands than a regular CC, even with limited computational capability. Various challenges arise, such as task scheduling, resource allocation, and offloading, substantially influencing the overall performance. Nevertheless, most of the proposed solutions are characterized by non-convexity and complexity, as they enable continuous learning ability for the dynamic environment and make a real-time inference with low computational complexity. Applying AI to networking challenges has gained popularity over the past few decades. For instance, ML has been widely used in the networking domain due to its ability to interact with complex environments and make decisions. It may also provide eminent solutions to improve network performance in many subdomains, such as resource allocation, network traffic prediction and classification, congestion control, and routing [32].

In most UAV-enabled MEC architectures, a controller called the follow-me cloud (FMC) collects information about overall system states (i.e., users, UAVs, and MEC servers). This control unit is in charge of managing offloading activities using AI technologies. Figure 5 displays an example of a cloudlet mounted on a UAV for a UAV-assisted MEC system. The UAV receives offloaded tasks from an IoT device and sends the results back upon fulfillment of the task's execution. Furthermore, the UAV could transport the received data to the nearest ground servers in case of complex processing requirements that overfit the onboard cloudlet's capabilities. The system may contain a set of UAVs that serve a massive area of deployed IoT devices that include mobile phones, sensors, vehicles, and robots. The data generated by the users is analyzed and treated via onboard cloudlets with the help of the AI approach.

As shown in Table 5, the DRL, DL, and GA can be utilized to solve task offloading challenges such as energy consumption limits, processing, and latency. Furthermore, for resource allocation management issues, the algorithms RL, DRL, GA, and RL-ACO might be utilized to regulate the energy consumption in UAVs and reduce the latency and reaction time. Moreover, the later algorithms, DE and ACO, can be applied to address joint optimization difficulties, whereas DL may enhance UAVs' security and path planning performance.

MEC networks should offer seamless connection, meet the QoS needs of many sensors, and handle a vast volume of data created by physical surroundings. AI approaches offering robust analysis, learning, optimizing, and intelligent recognition capabilities may be integrated into UAV-enabled MEC systems to intelligently carry out performance optimization, information discovery, advanced learning, structure organization, and complex decisionmaking. Relevant AI techniques include supervised learning, unsupervised learning, and reinforcement learning. 


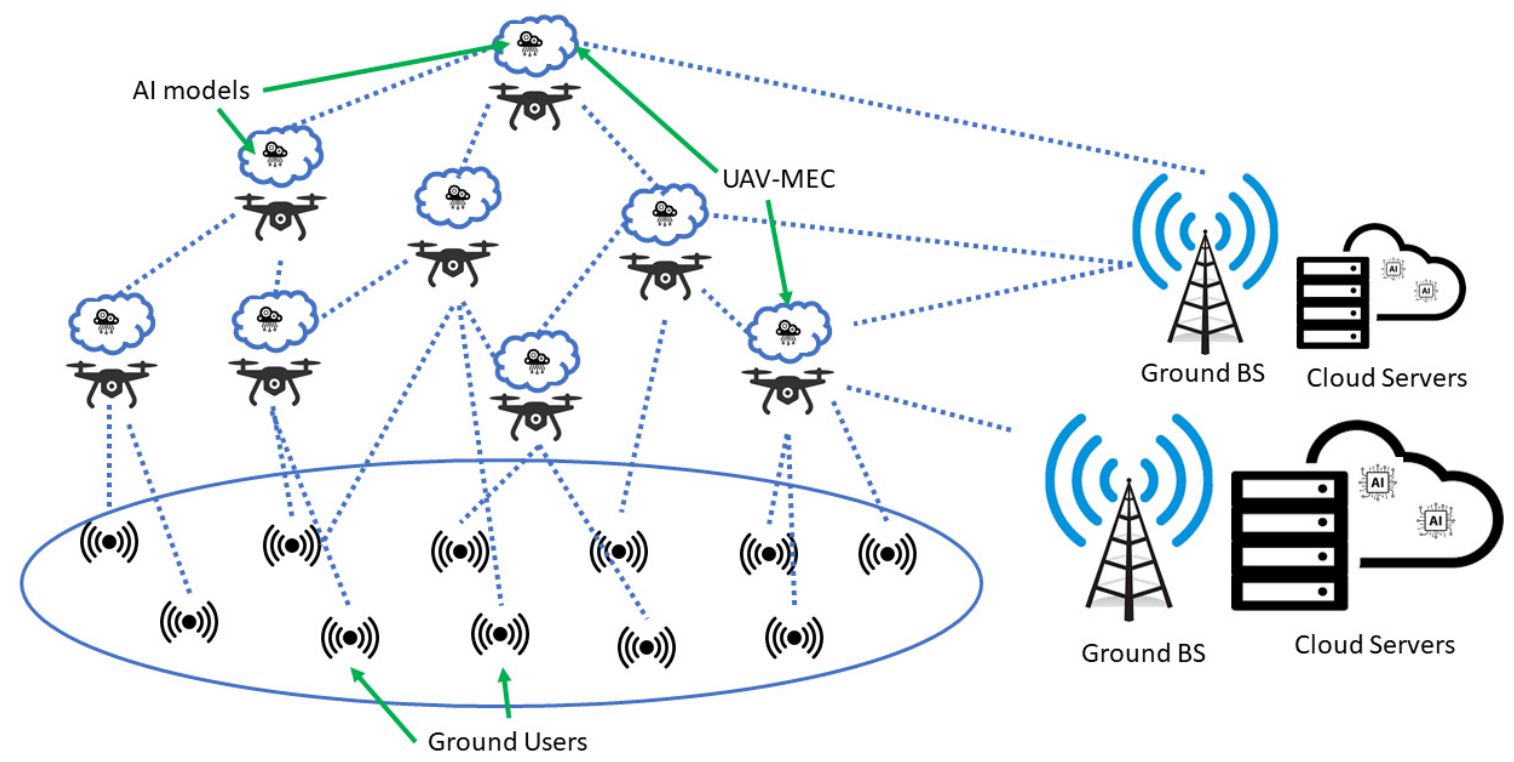

Figure 5. UAV-enabled MEC architecture.

For instance, DRL does not require labeled training data for resource allocation. Thus, it may enhance the process of offloading choices based on various states of the channel environment. This results in reducing the energy bandwidth and computations. To that end, an MDP method was provided to reduce the delay, energy consumption, and bandwidth in UAV-assisted MEC by analyzing the instability of energy arrival, stochastic computation tasks that are provided by wireless users, and a time-varying channel state [112]. According to the authors of [113], UAV-assisted MEC-based DL and DRL would ease the development of next-generation communications. This is particularly true for joint optimization challenges to improve system efficiency. Furthermore, UAV communication will be incorporated with $6 \mathrm{G}$ networks, where IoT device mobility might lead to position uncertainty. In this case, DRL, as a hybrid of DL and RL, can offer decision-making solutions and optimize handover issues in real-time. This approach allows UAVs to move dynamically and in real-time while assuring and minimizing communication delay [114].

Table 5. AI-based approaches used in UAV-enabled MEC.

\begin{tabular}{ccll}
\hline Addressed Issues & AI Approach & \multicolumn{1}{c}{ Metrics } & \multicolumn{1}{c}{ References } \\
\hline \multirow{5}{*}{ Task offloading } & RL & Energy consumption, processing time, latency & {$[115]$} \\
& DRL & Energy consumption, latency, cost & {$[116]$} \\
& GA & Energy consumption, latency & {$[117,118]$} \\
& DL & Security, privacy, task prediction, and & {$[119,120]$} \\
& computation offloading & {$[121]$} \\
\hline \multirow{5}{*}{ Resources allocation } & RL & Execution time & {$[122,123]$} \\
& Resources allocation, energy & {$[124,125]$} \\
& DRL & Latency, response time, resource utilization, & {$[126]$} \\
& energy consumption & {$[123]$} \\
\hline \multirow{5}{*}{ Joint optimization issue } & RL-ACO & Energy consumption, latency & {$[127]$} \\
& Throughput & {$[128]$} \\
& DRL & Security and privacy, energy consumption & {$[129]$} \\
& GA & Cost, energy consumption, latency & {$[130]$} \\
\hline Security & DL & Energy consumption, makespan & {$[131]$} \\
\hline Path planning & Energy consumption, cost & {$[127]$} \\
\hline & RL & Energy consumption & {$[35,132,133]$} \\
\hline
\end{tabular}


Authors in [134] have proved that the deep supervised learning (DSL) method minimizes the computation and offloading overheads and cost of mobile users in the MEC system. This technique allows terrestrial users to obtain an optimal offloading policy, with energy-saving and processing speed enhancement.

The authors of [91] have investigated the challenges associated with applying AI techniques to UAV-NOMA-MEC networks. They proposed an architecture that relies on AI-based approaches, particularly on federated learning and reinforcement learning. The proposed framework was dedicated to enabling terrestrial mobile users to offload their computational tasks simultaneously, intelligently, and flexibly, thus enhancing their connectivity and reducing their transmission latency and energy consumption.

The authors of [135] used RL and transfer learning algorithms to reduce the latency and energy consumption in UAV-enabled MEC networks. They proved that transfer learning, when combined with RL, could significantly enhance the system training performance when the users operated dynamically.

An optimization problem based on MDP was formulated by the authors of [35] to address UAV trajectory optimization in UAV-mounted MEC networks. The approach aimed to enhance the overall system QoS, maximize the long-term system reward, and meet the QoS constraint, including the computation tasks of mobile users. In [136], the authors have used the semi-Markov process and DRL-based algorithms to address the UAV-enabled MEC networks' offloading decisions and resource management policies.

The authors of [137] have developed a platform known as a flying UAV MEC, in which UAVs are outfitted with computing resources and provide task-offloading services to users. The suggested architecture's primary purpose was to maximize UAV aerial trajectory, user association, and resource allocation. The trajectory control method has been used to address the optimization issue, assisted by an RL-based technique. The results of this approach outperformed the results of the equivalent benchmark methods. Moreover, the authors of [138] formulated an ML-based novel framework to optimize UAV trajectories by estimating the user's mobility data to find optimal initial UAV placement locations. The joint problem, consisting of UAVs trajectories and power control to improve the sum rate and maintain the data rate requirements of mobile users, was also addressed in the employment of a multi-agent Q-learning-based technique. A smart offloading based on the deep Q-learning approach was proposed by the authors of [139] to optimize performance in terms of the delay perceived by ground devices connected to the FANET, to enable MEC in the $5 \mathrm{G}$ field.

\section{Discussion and Open Issues}

Computational intelligence, when combined with UAVs, has improved the underlying technologies and implementations of IoT applications. Nevertheless, researchers should address the remaining challenges. Therefore, some significant issues need more attention in order to benefit from UAVs for the realization of flying ECs. These issues may be related to scientific, technological, and societal problems, including challenges regarding the technical aspects of the technology, security and privacy concerns, and standardization. The challenges concern both the UAVs themselves and EC devices. Therefore, great effort is needed to address the following challenges.

Energy consumption: In any UAV-enabled IoT system, most sensors, edge devices, and drones are battery-powered and can only remain powered for a limited period. Therefore, efficient energy consumption protocols and mechanisms are essential for UAVs and IoT devices to ensure a long network lifetime. Improvement efforts should address the hardware and software architecture of the IoT components by integrating energy-harvesting solutions or efficient routing protocols. For example, AI schemes can be adopted to predict a UAV's residual energy and energy dissipation through the precise adjustment of transmission and control parameters concerning channel changes. The processing energy dissipation, communication energy, storage energy, and environmental reliability should be considered to mitigate the energy of UAVs $[115,140]$. Moreover, the issues listed below should also 
be considered to mitigate energy consumption, to extend the on-air flying time of the UAVs employed.

Interference: The communication links between UAVs and IoT devices may become disconnected due to interference, which reduces latency performance and may also affect the overall network, especially when the UAVs act as a mobile BS. Therefore, the problem of interference should be addressed, especially in urban and industrial environments, where the number of interconnected devices on the same frequency band is very dense. An AI-based solution can be applied in UAV-enabled MEC to predict the signal-to-interference ratio, based on the collected channel state information (CSI). It is then possible to decide on the most appropriate scheme to combat the impact of interference or to adjust transmission parameters in order to reduce power consumption.

Interoperability: In any IoT environment, many devices are expected to handle the required tasks without any effort from the end-users. These devices try to connect and interact with each other using different protocols and communication paradigms. With this diversity, it is sometimes challenging to orchestrate the system smoothly. Therefore, further investigations are necessary to unify the controller and communication protocols to ensure interoperability among them.

Mobility and path planning: UAVs require auto-maneuvering without distant human pilot intervention in random dynamic IoT applications. Therefore, many intelligent methods should be considered to enable autonomous UAV flights that include collision avoidance. AI algorithms are the most viable solutions that can be used to detect obstacles and avoid collisions [141]. For instance, DL techniques can enable learning from the gathered data, which makes their integration into UAV a significant solution. A serious effort should be considered to develop green-federated AI solutions, such as RL, to ensure accurate auto-flying decisions and path and trajectory planning [35,132].

Scalability: The scalability challenges concern resources, applications, load balancing, and connections. Enabling high scalability requires designing efficient schemes that could effectively manage heterogeneous mobile devices and servers in a UAV-enabled MEC environment, with its highly dynamic demand behavior. For example, some mobile applications, such as UAVs and autonomous driving, require high data throughput offloading to successfully provide such services, despite heterogeneity in the number of mobile devices and the dynamic demand behavior of the applications mentioned in the MEC environment. The MEC system should have an acceptable degree of scalability regarding the number of servers and services required. As a result, it is challenging for difficult areas to establish connections and communications between mobile devices and servers. To achieve convenient and cost-effective mobility and scalability, resulting in continuous service, it is necessary to have a stable connection, even in adverse environmental conditions.

Security: Drones enable ubiquitous services on an IoT scale. The communications and controls are realized through P2P wireless transmissions and thus are open to many security threats. Therefore, unauthorized connections threaten the system's privacy. It is necessary to propose and design security mechanisms to improve UAV-enabled MEC systems' security [142]. The effort should focus on the physical, application, and MAC layers. The solutions should integrate recent robust techniques such as blockchains, especially in IoT applications where UAVs are expected to facilitate communication networks. The combination of UAVs with blockchain and AI is now an exciting avenue of research to predict all sorts of attacks and malware that threaten the system's privacy [127]. However, while there has been significant progress in this area, more work needs to be done. Power consumption might be a critical constraint for blockchain-based applications as they need more computing power. Increasingly complex attacks, such as quantum attacks, ML, and theory-based game attacks, could challenge the privacy of blockchain systems [143]. More research is needed to make private blockchain networks more immutable and secure in UAV-enabled MEC architectures. Moreover, blockchain-enabled softwarization for secure UAV network systems is also a critical challenge. Blockchain implementation in UAV 
systems presents a complex system integration problem requiring rigorous testing before concrete implementation [12].

Integration of AI schemes: Recently, the utilization of AI schemes among DL, ML, and RL has become increasingly of interest, to benefit from their powerful performance. AI schemes are being integrated into UAV-enabled MEC systems as a way to make contextual decisions and learn from aggregated data gathered from previous tracking experiences. The data collected by the sensors in real-time can be profitably exploited for the autonomous piloting of UAVs. Therefore, processing tasks demand strict computing and storage requirements at computing facilities, to be executed exceptionally at the edge. UAVs also have energy and storage limitations; thus, portable and lightweight RL, ML, and DL algorithms should be designed to cope with these constraints.

Regulations: Despite the rapid emergence of this technology, UAV regulations are still in their infancy, and a lack of heterogeneity of national rules and varying levels of implementation can be observed. These regulations differ from state to state, and the type of regulation is based on the requirements of that use. Several problems are associated with UAV regulation, including delays in flight approval and poorly documented administrative processes limiting the desired flexibility, preventing widespread use of the technology [144].

Latency and computation issues: Communication latency and computing optimization remain concerns in MEC-enabled UAV applications, affecting transmission efficiency and reaction time under communication and computing constraints. UAV transmission success may be accomplished by offering effective methods that decrease response delay. In one study [145], for example, stochastic geometry and queueing theory approaches were studied to minimize the response delay for a MEC-enabled UAV network.

Resource allocation: Due to UAV battery concerns and trajectory constraints, resource allocation is critical in UAV-enabled MEC networks. Resources allocation is involved in three processes: computing task offloading, local computing, and UAV hovering. Designing an appropriate trajectory can ultimately compromise the calculated performance and operating costs. According to the authors of [146], resource allocation may be structured to meet various goals, including computing bit maximization, energy minimization, computational efficiency maximization, cost minimization, completion time minimization, and the consideration of fairness.

UAV-enabled IoT for B5G and 6G issues: Data-gathering faces several problems in IoT that extend beyond the fifth-generation (B5G) and upcoming sixth-generation (6G) wireless mobile networks. A large amount of data will be generated by heterogeneous devices, particularly IoT devices and smartphones at the mobile edge; these data are fragmented and dispersed among several machines. Therefore, distributed solutions such as UAV-enabled MEC should be leveraged near mobile users to offload and process the data. Meanwhile, growing privacy concerns make data collecting increasingly challenging. This consumes more energy and decreases the system's offloading time [147].

\section{Conclusions}

The development of wireless communication technologies and IT services has made the role of drones more ubiquitous in various fields of application. Drones can serve as a link between smart devices and cloud data centers, to provide many services that can increase the performance of a given IoT system. To the best of our knowledge, this paper is considered the first work that provides a review of drone-enabled MECs based on AI. This work discusses the current research trends and provides future insights into the potential uses of UAV-enabled MEC in the field of IoT. It highlights the potential roles that UAVs can play in enabling communications and intelligent computing in the IoT era, by referring to the most widely emerging AI techniques that are employed. As mentioned, UAVs as MECs are attracting considerable interest in scientific research, to address the various problems and challenges experienced in order to improve the performance of such applications in different scenarios, such as resource allocation, energy efficiency, latency, task offloading, and security. UAV-enabled MEC present an emerging concept that require 
more effort to achieve the necessary performance in every scenario of UAV application in IoT environments, especially regarding MEC.

Author Contributions: Y.Y. carried out this research as part of his Ph.D. thesis, under the supervision of A.E.O. and M.A. Y.Y., I.E.-Z., A.G.-G., M.A. and A.E.O. contributed to the design and implementation of the research review, upon the analysis and the selection of the papers, and the writing of the manuscript. All authors have read and agreed to the published version of the manuscript.

Funding: This research received no external funding.

Institutional Review Board Statement: Not applicable.

Informed Consent Statement: Not applicable.

Conflicts of Interest: The authors declare no conflict of interest.

\section{Abbreviations}

The following abbreviations are used in this manuscript.

$\begin{array}{ll}\text { Abbreviation } & \text { Definition } \\ \text { AI } & \text { Artificial intelligence } \\ \text { CC } & \text { Cloud computing } \\ \text { CPU } & \text { Central processing units } \\ \text { CV } & \text { Computer vision } \\ \text { DRL } & \text { Deep reinforcement learning } \\ \text { EC } & \text { Edge computing } \\ \text { FANET } & \text { Flying ad hoc network } \\ \text { FI } & \text { Fuzzy inference } \\ \text { GA } & \text { Genetic algorithm } \\ \text { GPU } & \text { Graphics processing units } \\ \text { IoFT } & \text { Internet of Flying Things } \\ \text { IoT } & \text { Internet of Things } \\ \text { ISM } & \text { Industrial scientific medical } \\ \text { LC } & \text { Local computing } \\ \text { LoRa } & \text { Long-range } \\ \text { TDMA } & \text { Time division multiple access } \\ \text { MEC } & \text { Mobile edge computing } \\ \text { ML } & \text { Machine learning } \\ \text { NOMA } & \text { Non-orthogonal multiple access } \\ \text { QoS } & \text { Quality of service } \\ \text { RL } & \text { Reinforcement learning } \\ \text { UAV } & \text { Unmanned aerial vehicle } \\ \text { VANET } & \text { Vehicular ad hoc network } \\ \text { BLE } & \text { Bluetooth low energy } \\ \text { RL-ACO } & \text { Reinforcement learning based on ant-colony optimization } \\ \text { DRL } & \text { Deep reinforcement learning } \\ \text { VTOL } & \text { Vertical take-off and landing } \\ \text { HTOL } & \text { Horizontal take-off and landing } \\ \text { LiDAR } & \text { Light detection and ranging } \\ & \end{array}$

\section{References}

1. Nižetić, S.; Šolić, P.; López-de-Ipiña González-de-Artaza, D.; Patrono, L. Internet of Things (IoT): Opportunities, issues and challenges towards a smart and sustainable future. J. Clean. Prod. 2020, 274, 122877. [CrossRef]

2. Shafique, K.; Khawaja, B.A.; Sabir, F.; Qazi, S.; Mustaqim, M. Internet of things (IoT) for next-generation smart systems: A review of current challenges, future trends and prospects for emerging 5G-IoT Scenarios. IEEE Access 2020, 8, 23022-23040. [CrossRef]

3. Mozaffari, M.; Saad, W.; Bennis, M.; Nam, Y.H.; Debbah, M. A Tutorial on UAVs for Wireless Networks: Applications, Challenges, and Open Problems. IEEE Commun. Surv. Tutor. 2019, 21, 2334-2360. [CrossRef]

4. Lyu, J.; Zeng, Y.; Zhang, R.; Lim, T.J. Placement Optimization of UAV-Mounted Mobile Base Stations. IEEE Commun. Lett. 2017, 21, 604-607. [CrossRef] 
5. Alzenad, M.; El-Keyi, A.; Lagum, F.; Yanikomeroglu, H. 3-D Placement of an Unmanned Aerial Vehicle Base Station (UAV-BS) for Energy-Efficient Maximal Coverage. IEEE Wirel. Commun. Lett. 2017, 6, 434-437. [CrossRef]

6. Zhang, S.; Zhang, H.; Di, B.; Song, L. Joint trajectory and power optimization for UAV sensing over cellular networks. IEEE Commun. Lett. 2018, 22, 2382-2385. [CrossRef]

7. Motlagh, N.H.; Bagaa, M.; Taleb, T. UAV-Based IoT Platform: A Crowd Surveillance Use Case. IEEE Commun. Mag. 2017, 55, 128-134. [CrossRef]

8. Chen, W.; Liu, B.; Huang, H.; Guo, S.; Zheng, Z. When UAV Swarm Meets Edge-Cloud Computing: The QoS Perspective. IEEE Netw. 2019, 33, 36-43. [CrossRef]

9. Mao, Y.; You, C.; Zhang, J.; Huang, K.; Letaief, K.B. A Survey on Mobile Edge Computing: The Communication Perspective. IEEE Commun. Surv. Tutor. 2017, 19, 2322-2358. [CrossRef]

10. Cheng, N.; Xu, W.; Shi, W.; Zhou, Y.; Lu, N.; Zhou, H.; Shen, X. Air-ground integrated mobile edge networks: Architecture, challenges, and opportunities. IEEE Commun. Mag. 2018, 56, 26-32. [CrossRef]

11. Liu, J.; Zhou, A.; Liu, C.; Zhang, T.; Qi, L.; Wang, S.; Buyya, R. Reliability-Enhanced Task Offloading in Mobile Edge Computing Environments. IEEE Internet Things J. 2021, 124-129. [CrossRef]

12. Kumari, A.; Gupta, R.; Tanwar, S.; Kumar, N. A taxonomy of blockchain-enabled softwarization for secure UAV network. Comput. Commun. 2020, 161, 304-323. [CrossRef]

13. Mehta, P.; Gupta, R.; Tanwar, S. Blockchain envisioned UAV networks: Challenges, solutions, and comparisons. Comput. Commun. 2020, 151, 518-538. [CrossRef]

14. Abrar, M.; Ajmal, U.; Almohaimeed, Z.M.; Gui, X.; Akram, R.; Masroor, R. Energy Efficient UAV-Enabled Mobile Edge Computing for IoT Devices: A Review. IEEE Access 2021, 9, 127779-127798. [CrossRef]

15. Sharma, A.; Vanjani, P.; Paliwal, N.; Basnayaka, C.M.W.; Jayakody, D.N.K.; Wang, H.C.; Muthuchidambaranathan, P. Communication and networking technologies for UAVs: A survey. J. Netw. Comput. Appl. 2020, 168, 102739. [CrossRef]

16. Zhang, W.; Li, L.; Zhang, N.; Han, T.; Wang, S. Air-Ground Integrated Mobile Edge Networks: A Survey. IEEE Access 2020, 8, 125998-126018. [CrossRef]

17. Shakarami, A.; Ghobaei-Arani, M.; Shahidinejad, A. A survey on the computation offloading approaches in mobile edge computing: A machine learning-based perspective. Comput. Netw. 2020, 182, 107496. [CrossRef]

18. Korchenko, A.G.; Illyash, O.S. The generalized classification of Unmanned Air Vehicles. In Proceedings of the 2013 IEEE 2nd International Conference Actual Problems of Unmanned Air Vehicles Developments Proceedings (APUAVD), Kiev, Ukraine, 15-17 October 2013; pp. 28-34. [CrossRef]

19. Chamola, V.; Kotesh, P.; Agarwal, A.; Naren; Gupta, N.; Guizani, M. A Comprehensive Review of Unmanned Aerial Vehicle Attacks and Neutralization Techniques. Ad Hoc Netw. 2021, 111, 102324. [CrossRef]

20. Guo, Y.; Yin, G.; Sun, H.; Wang, H.; Chen, S.; Senthilnath, J.; Wang, J.; Fu, Y. Scaling effects on chlorophyll content estimations with RGB camera mounted on a UAV platform using machine-learning methods. Sensors 2020, 20, 5130. [CrossRef]

21. Lin, Y.C.; Cheng, Y.T.; Zhou, T.; Ravi, R.; Hasheminasab, S.M.; Flatt, J.E.; Troy, C.; Habib, A. Evaluation of UAV LiDAR for mapping coastal environments. Remote Sens. 2019, 11, 2893. [CrossRef]

22. Miyoshi, G.T.; Arruda, S.; Osco, L.P.; Junior, M.; Gonçalves, D.N.; Imai, N.N. A Novel Deep Learning Method to Identify Single Tree Species in UAV-Based Hyperspectral Images. Remote Sens. 2020, 12, 1294. [CrossRef]

23. Cen, H.; Wan, L.; Zhu, J.; Li, Y.; Li, X.; Zhu, Y.; Weng, H. Dynamic monitoring of biomass of rice under different nitrogen treatments using a lightweight UAV with dual image-Frame snapshot cameras. Plant Methods 2019, 15, 32. [CrossRef] [PubMed]

24. Hill, A.C.; Laugier, E.J. Archaeological Remote Sensing Using Infrared (NIR) Imagery: A Case Study at the Enfield Shaker Village, New Hampshire. Remote Sens. 2020, 12, 690. [CrossRef]

25. Zaidi, S.; Atiquzzaman, M.; Calafate, C.T. Internet of Flying Things (IoFT): A Survey. Comput. Commun. 2021, 165, 53-74. [CrossRef]

26. Nazib, R.A.; Moh, S. Routing Protocols for Unmanned Aerial Vehicle-Aided Vehicular Ad Hoc Networks: A Survey. IEEE Access 2020, 8, 77535-77560. [CrossRef]

27. Al-Fuqaha, A.; Guizani, M.; Mohammadi, M.; Aledhari, M.; Ayyash, M. Internet of Things: A Survey on Enabling Technologies, Protocols, and Applications. IEEE Commun. Surv. Tutor. 2015, 17, 2347-2376. [CrossRef]

28. Mekki, K.; Bajic, E.; Chaxel, F.; Meyer, F. A comparative study of LPWAN technologies for large-scale IoT deployment. ICT Express 2019, 5, 1-7. [CrossRef]

29. Yazid, Y.; Ez-Zazi, I.; Arioua, M.; Oualkadi, A.E.L. On the LoRa performances under different physical layer parameter selection. In Proceedings of the 2020 International Symposium on Advanced Electrical and Communication Technologies (ISAECT), Kenitra, Morocco, 25-27 November 2020; pp. 13-18. [CrossRef]

30. Akpakwu, G.A.; Silva, B.J.; Hancke, G.P.; Abu-Mahfouz, A.M. A Survey on 5G Networks for the Internet of Things: Communication Technologies and Challenges. IEEE Access 2017, 6, 3619-3647. [CrossRef]

31. Mignardi, S.; Marini, R.; Verdone, R.; Buratti, C. On the performance of a uav-aided wireless network based on nb-iot. Drones 2021, 5, 94. [CrossRef]

32. Erdelj, M.; Król, M.; Natalizio, E. Wireless Sensor Networks and Multi-UAV systems for natural disaster management. Comput. Netw. 2017, 124, 72-86. [CrossRef] 
33. Sohail, M.F.; Member, S. Non-Orthogonal Multiple Access for Unmanned Aerial Vehicle Assisted Communication. IEEE Access 2018, 6, 22716-22727. [CrossRef]

34. Liu, D.; Xu, Y.; Wang, J.; Chen, J.; Yao, K.; Wu, Q.; Anpalagan, A. Opportunistic UAV utilization in wireless networks: Motivations, applications, and challenges. IEEE Commun. Mag. 2020, 58, 62-68. [CrossRef]

35. Liu, Q.; Shi, L.; Sun, L.; Li, J.; Ding, M.; Shu, F.S. Path Planning for UAV-Mounted Mobile Edge Computing with Deep Reinforcement Learning. IEEE Trans. Veh. Technol. 2020, 69, 5723-5728. [CrossRef]

36. Ali, N.; Jhanjhi, N.Z.; Nawaz, S.; Sher, R.; Usmani, A. Smart traffic monitoring system using Unmanned Aerial Vehicles (UAVs). Comput. Commun. 2020, 157, 434-443. [CrossRef]

37. Jiang, F.; Wang, K.; Dong, L.; Pan, C.; Xu, W.; Yang, K. Deep Learning Based Joint Resource Scheduling Algorithms for Hybrid MEC Networks. IEEE Internet Things J. 2020, 7, 6252-6265. [CrossRef]

38. Zhou, F.; Wu, Y.; Sun, H.; Chu, Z. UAV-Enabled mobile edge computing: Offloading optimization and trajectory design. In Proceedings of the 2018 IEEE International Conference on Communications (ICC), Kansas City, MO, USA, 20-24 May 2018; pp. 1-6. [CrossRef]

39. Kitpo, N.; Inoue, M. Early rice disease detection and position mapping system using drone and IoT architecture. In Proceedings of the 2018 12th South East Asian Technical University Consortium (SEATUC), Yogyakarta, Indonesia, 12-13 March 2018. [CrossRef]

40. Popescu, D.; Stoican, F.; Stamatescu, G.; Ichim, L.; Dragana, C. Advanced UAV-WSN System for Intelligent Monitoring in Precision Agriculture. Sensors 2020, 20, 817. [CrossRef]

41. Mazzia, V.; Comba, L.; Khaliq, A.; Chiaberge, M.; Gay, P. UAV and Machine Learning Based Refinement of a Satellite-Driven Vegetation Index for Precision Agriculture. Sensors 2020, 20, 2530. [CrossRef]

42. Maddikunta, P.K.R.; Hakak, S.; Alazab, M.; Bhattacharya, S.; Gadekallu, T.R.; Khan, W.Z.; Pham, Q.V. Unmanned Aerial Vehicles in Smart Agriculture: Applications, Requirements, and Challenges. IEEE Sens. J. 2021, 21, 17608-17619. [CrossRef]

43. Grosso, R.; Mecca, U.; Moglia, G.; Prizzon, F.; Rebaudengo, M. Collecting built environment information using UAVs: Time and applicability in building inspection activities. Sustainability 2020, 12, 4731. [CrossRef]

44. Panday, U.S.; Shrestha, N.; Maharjan, S.; Pratihast, A.K.; Shahnawaz; Shrestha, K.L.; Aryal, J. Correlating the plant height of wheat with aboveground biomass and crop yield using drone imagery and crop surface model, a case study from Nepal. Drones 2020, 4, 28. [CrossRef]

45. Hentschke, M.; de Freitas, E.P.; Hennig, C.H.; da Veiga, I.C.G. Evaluation of altitude sensors for a crop spraying drone. Drones 2018, 2, 25. [CrossRef]

46. Suen, H.K.; Ary, D. Reliability: Conventional Methods. Anal. Quant. Behav. Obs. Data 2020, 111-142. [CrossRef]

47. Agapiou, A. Vegetation extraction using visible-bands from openly licensed unmanned aerial vehicle imagery. Drones 2020, 4, 27. [CrossRef]

48. Sharma, V.; Choudhary, G.; Ko, Y. Behavior and Vulnerability Assessment of Drones-Enabled Industrial Internet of Things (IIoT). IEEE Access 2018, 6, 43368-43383. [CrossRef]

49. Salhaoui, M.; Guerrero-González, A.; Arioua, M.; Ortiz, F.J.; El Oualkadi, A.; Torregrosa, C.L. Smart industrial iot monitoring and control system based on UAV and cloud computing applied to a concrete plant. Sensors 2019, 19, 3316. [CrossRef] [PubMed]

50. Nooralishahi, P.; Ibarra-Castanedo, C.; Deane, S.; López, F.; Pant, S.; Genest, M.; Avdelidis, N.P.; V Maldague, X.P.; González Jorge, H.; Miguel González de Santos, L. Drone-Based Non-Destructive Inspection of Industrial Sites: A Review and Case Studies. Drones 2021, 5, 106. [CrossRef]

51. Shahmoradi, J.; Talebi, E.; Roghanchi, P.; Hassanalian, M. A Comprehensive Review of Applications of Drone Technology in the Mining Industry. Drones 2020, 4, 34. [CrossRef]

52. Cowley, D.C.; Moriarty, C.; Geddes, G.; Brown, G.L.; Wade, T.; Nichol, C.J. UAVs in context: Archaeological airborne recording in a national body of survey and record. Drones 2018, 2, 2. [CrossRef]

53. Mangewa, L.J.; Ndakidemi, P.A.; Munishi, L.K. Integrating UAV technology in an ecological monitoring system for community wildlife management areas in Tanzania. Sustainability 2019, 11, 6116. [CrossRef]

54. Rees, A.F.; Avens, L.; Ballorain, K.; Bevan, E.; Broderick, A.C.; Carthy, R.R.; Christianen, M.J.A.; Duclos, G.; Heithaus, M.R.; Johnston, D.W.; et al. The potential of unmanned aerial systems for sea turtle research and conservation: A review and future directions. Endanger. Species Res. 2018, 35, 81-100. [CrossRef]

55. Salhaoui, M.; Molina-molina, J.C.; Guerrero-gonz, A.; Arioua, M.; Ortiz, F.J. Autonomous Underwater Monitoring System for Detecting Life on the Seabed by Means of Computer Vision Cloud Services. Remote Sens. 2020, 12, 198. [CrossRef]

56. Wang, D.; Shao, Q.; Yue, H. Surveying wild animals from satellites, manned aircraft and unmanned aerial systems (UASs): A review. Remote Sens. 2019, 11, 1308. [CrossRef]

57. Chamola, V.; Hassija, V.; Gupta, V.; Guizani, M. A Comprehensive Review of the COVID-19 Pandemic and the Role of IoT, Drones, AI, Blockchain, and 5G in Managing its Impact. IEEE Access 2020, 8, 90225-90265. [CrossRef]

58. Jeyabalan, V.; Nouvet, E.; Meier, P.; Donelle, L. Context-specific challenges, opportunities, and ethics of drones for healthcare delivery in the eyes of program managers and field staff: A multi-site qualitative study. Drones 2020, 4, 44. [CrossRef]

59. Zhu, J.; Sun, K.; Jia, S.; Li, Q.; Hou, X.; Lin, W.; Liu, B.; Qiu, G. Urban Traffic Density Estimation Based on Neural Network. IEEE J. Sel. Top. Appl. Earth Obs. Remote Sens. 2018, 11, 4968-4981. [CrossRef]

60. Congress, S.S.C.; Puppala, A.J.; Lundberg, C.L. Total system error analysis of UAV-CRP technology for monitoring transportation infrastructure assets. Eng. Geol. 2018, 247, 104-116. [CrossRef] 
61. Outay, F.; Abdullah, H.; Adnan, M. Applications of unmanned aerial vehicle (UAV) in road safety, traffic and highway infrastructure management: Recent advances and challenges. Transp. Res. Part A 2020, 141, 116-129. [CrossRef]

62. Malihi, S.; Javad, M.; Zoej, V.; Hahn, M. Large-Scale Accurate Reconstruction of Buildings Employing Point Clouds Generated from UAV Imagery. Remote Sens. 2018, 10, 1148. [CrossRef]

63. Mohamed, N.; Al-jaroodi, J.; Jawhar, I.; Idries, A.; Mohammed, F. Technological Forecasting \& Social Change Unmanned aerial vehicles applications in future smart cities. Technol. Forecast. Soc. Chang. 2020, 153, 119293. [CrossRef]

64. Qi, F.; Zhu, X.; Mang, G.; Kadoch, M.; Li, W. Internet of things for smart citites: Technologies and applications UAV Network and IoT in the Sky for Future Smart Cities. IEEE Netw. 2019, 33, 96-101. [CrossRef]

65. Orgeira-crespo, P.; Ulloa, C.; Rey-gonzalez, G.; Antonio, P. Methodology for Indoor Positioning and Landing of an Unmanned Aerial Vehicle in a Smart Manufacturing Plant for Light Part Delivery. Electronics 2020, 9, 1680. [CrossRef]

66. Erdelj, M.; Natalizio, E. UAV-assisted disaster management: Applications and open issues. In Proceedings of the 2016 International Conference on Computing, Networking and Communications (ICNC), Kauai, HI, USA, 15-18 February 2016; pp. 1-5. [CrossRef]

67. Erdelj, M.; Natalizio, E.; Chowdhury, K.R.; Akyildiz, I.F. Help from the Sky: Leveraging UAVs for Disaster Management. IEEE Pervasive Comput. 2017, 16, 24-32. [CrossRef]

68. Liu, D.; Xia, X.; Chen, J.; Li, S. Integrating Building Information Model and Augmented Reality for Drone-Based Building Inspection. J. Comput. Civ. Eng. 2021, 35, 04020073. [CrossRef]

69. Chen, S.; Laefer, D.F.; Mangina, E.; Zolanvari, S.M.I.; Byrne, J. UAV Bridge Inspection through Evaluated 3D Reconstructions. J. Bridg. Eng. 2019, 24, 05019001. [CrossRef]

70. Hildmann, H.; Kovacs, E. Review: Using Unmanned Aerial Vehicles (UAVs) as Mobile Sensing Platforms (MSPs) for Disaster Response, Civil Security and Public Safety. Drones 2019, 3, 59. [CrossRef]

71. Bang, S.; Kim, H.; Kim, H. Automation in Construction UAV-based automatic generation of high-resolution panorama at a construction site with a focus on preprocessing for image stitching. Autom. Constr. 2017, 84, 70-80. [CrossRef]

72. Lin, X.; Yajnanarayana, V.; Muruganathan, S.D.; Gao, S.; Asplund, H.; Maattanen, H.L.; Bergstrom, M.; Euler, S.; Wang, Y.P.E. The Sky is Not the Limit: LTE for Unmanned Aerial Vehicles. IEEE Commun. Mag. 2018, 56, 204-210. [CrossRef]

73. Villa, D.K.D.; Brandão, A.S.; Sarcinelli-Filho, M. A Survey on Load Transportation Using Multirotor UAVs. J. Intell. Robot. Syst. Theory Appl. 2020, 98, 267-296. [CrossRef]

74. Alladi, T.; Chamola, V.; Sahu, N.; Guizani, M. Applications of blockchain in unmanned aerial vehicles: A review. Veh. Commun. 2020, 23, 100249. [CrossRef]

75. Ren, J.; Guo, H.; Xu, C.; Zhang, Y. Serving at the Edge: A Scalable IoT Architecture Based on Transparent Computing. IEEE Netw. 2017, 31, 96-105. [CrossRef]

76. Mach, P.; Becvar, Z. Mobile Edge Computing: A Survey on Architecture and Computation Offloading. IEEE Commun. Surv. Tutor. 2017, 19, 1628-1656. [CrossRef]

77. Lu, W.; Ding, Y.; Gao, Y.; Hu, S.; Wu, Y.; Zhao, N.; Gong, Y. Resource and Trajectory Optimization for Secure Communications in Dual-UAV-MEC Systems. IEEE Trans. Ind. Inform. 2021, 3203, 1-10. [CrossRef]

78. Chen, J.; Chen, S.; Luo, S.; Wang, Q.; Cao, B.; Li, X. An intelligent task of fl oading algorithm (iTOA) for UAV edge computing network. Digit. Commun. Netw. 2020, 6, 433-443. [CrossRef]

79. Zhu, S.; Lin, G.; Zhao, D.; Cheng, N.; Zhang, Q.; Lang, X. Learning-Based Computation Offloading Approaches in UAVs-Assisted Edge Computing. IEEE Trans. Veh. Technol. 2021, 70, 928-944. [CrossRef]

80. Liyanage, M.; Dar, F.; Sharma, R.; Flores, H. GEESE: Edge computing enabled by UAVs. Pervasive Mob. Comput. 2021, 72, 101340. [CrossRef]

81. Xu, J.; Liu, X.; Li, X.; Zhang, L.; Jin, J.; Yang, Y. Energy aware Computation Management Strategy for Smart Logistic System with MEC. IEEE Internet Things J. 2021, 4662, 1-17. [CrossRef]

82. Li, W.T.; Zhao, M.; Wu, Y.H.; Yu, J.J.; Bao, L.Y.; Yang, H.; Liu, D. Collaborative offloading for UAV—enabled time—sensitive MEC networks. EURASIP J. Wirel. Commun. Netw. 2021, 2021, 1. [CrossRef]

83. Liu, Y.; Xiong, K.; Ni, Q.; Member, S.; Fan, P.; Member, S.; Letaief, K.B. UAV-assisted Wireless Powered Cooperative Mobile Edge Computing: Joint Offloading, CPU Control and Trajectory Optimization. IEEE Internet Things J. 2019, 7, 2777-2790. [CrossRef]

84. Zhang, T.; Xu, Y.; Loo, J.; Yang, D.; Xiao, L. Joint Computation and Communication Design for UAV-Assisted Mobile Edge Computing in IoT. IEEE Trans. Ind. Inform. 2019, 16, 5505-5516. [CrossRef]

85. Hu, X.; Wong, K.K.; Yang, K.; Zheng, Z. UAV-Assisted Relaying and Edge Computing: Scheduling and Trajectory Optimization. IEEE Trans. Wirel. Commun. 2019, 18, 4738-4752. [CrossRef]

86. Du, Y.; Yang, K.; Wang, K.; Zhang, G.; Zhao, Y.; Chen, D. Joint Resources and Workflow Scheduling in UAV-Enabled WirelesslyPowered MEC for IoT Systems. IEEE Trans. Veh. Technol. 2019, 68, 10187-10200. [CrossRef]

87. Wang, Y.; Ru, Z.Y.; Wang, K.; Huang, P.Q. Joint Deployment and Task Scheduling Optimization for Large-Scale Mobile Users in Multi-UAV-Enabled Mobile Edge Computing. IEEE Trans. Cybern. 2020, 50, 3984-3997. [CrossRef] [PubMed]

88. Luo, Y.; Ding, W.; Zhang, B. Optimization of Task Scheduling and Dynamic Service Strategy for Multi-UAV-enabled Mobile Edge Computing System. IEEE Trans. Cogn. Commun. Netw. 2021, 7, 970-984. [CrossRef]

89. Ouyang, W.; Chen, Z.; Wu, J.; Yu, G.; Zhang, H. Dynamic Task Migration Combining Energy Efficiency and Load Balancing Optimization in Three-Tier UAV-Enabled Mobile Edge Computing System. Electronics 2021, 10, 190. [CrossRef] 
90. Guo, H.; Liu, J. UAV-Enhanced Intelligent Offloading for Internet of Things at the Edge. IEEE Trans. Ind. Inform. 2020, 16, 2737-2746. [CrossRef]

91. Zhang, B.; Zhang, G.; Ma, S.; Yang, K.; Wang, K. Efficient Multitask Scheduling for Completion Time Minimization in UAVAssisted Mobile Edge Computing. Mob. Inf. Syst. 2020, 2020, 8791030. [CrossRef]

92. Zhou, Y.; Pan, C.; Yeoh, P.L.; Wang, K.; Elkashlan, M.; Vucetic, B.; Li, Y. Secure Communications for UAV-Enabled Mobile Edge Computing Systems. IEEE Trans. Commun. 2020, 68, 376-388. [CrossRef]

93. Zhou, Z.; Feng, J.; Tan, L.; He, Y.; Gong, J. An air-ground integration approach for mobile edge computing in IoT. IEEE Commun. Mag. 2018, 56, 40-47. [CrossRef]

94. Narang, M.; Xiang, S.; Liu, W.; Gutierrez, J.; Chiaraviglio, L. UAV-assisted Edge Infrastructure for Challenged Networks. In Proceedings of the 2017 IEEE Conference on Computer Communications Workshops (INFOCOM WKSHPS), Atlanta, GA, USA, 1-4 May 2017. [CrossRef]

95. Hu, Q.; Cai, Y.; Yu, G.; Qin, Z.; Zhao, M.; Li, G.Y. Joint offloading and trajectory design for UAV-enabled mobile edge computing systems. IEEE Internet Things J. 2019, 6, 1879-1892. [CrossRef]

96. Chang, H.; Chen, Y.; Zhang, B.; Doermann, D. Multi-UAV Mobile Edge Computing and Path Planning Platform Based on Reinforcement Learning. IEEE Trans. Emerg. Top. Comput. Intell. 2021, 1-10. [CrossRef]

97. Diao, X.; Zheng, J.; Wu, Y.; Cai, Y.; Anpalagan, A. Joint Trajectory Design, Task Data, and Computing Resource Allocations for NOMA-Based and UAV-Assisted Mobile Edge Computing. IEEE Access 2019, 7, 117448-117459. [CrossRef]

98. Zhou, F.; Wu, Y.; Hu, R.Q.; Qian, Y. Computation rate maximization in UAV-Enabled wireless-powered mobile-edge computing systems. IEEE J. Sel. Areas Commun. 2018, 36, 1927-1941. [CrossRef]

99. Balamuralidhar, N.; Tilon, S.; Nex, F. MultEYE: Monitoring System for Real-Time Vehicle Detection, Tracking and Speed Estimation from UAV Imagery on Edge-Computing Platforms. Remote Sens. 2021, 13, 573. [CrossRef]

100. Yang, M.; Boubin, J.G.; Ping, H.; Tseng, H.; Hsu, Y.; Stewart, C.C. Adaptive autonomous UAV scouting for rice lodging assessment using edge computing with deep learning EDANet. Comput. Electron. Agric. 2020, 179, 105817. [CrossRef]

101. Yang, Z.; Chen, M.; Liu, X.; Liu, Y.; Chen, Y.; Cui, S.; Poor, H.V. Aerial Computing: Drones for Multi-Access Edge Computing AI-Driven UAV-NOMA-MEC in Next Generation Wireless Networks. IEEE Wirel. Commun. 2021, 28. [CrossRef]

102. Grasso, C.; Schembra, G. A Fleet of MEC UAVs to Extend a 5G Network Slice for Video Monitoring with Low-Latency Constraints. J. Sens. Actuator Netw. 2019, 8, 3. [CrossRef]

103. Nie, Y.; Member, S.; Zhao, J.; Member, S.; Gao, F.; Yu, F.R. Semi-Distributed Resource Management in UAV-Aided MEC Systems: A Multi-Agent Federated Reinforcement Learning Approach. IEEE Trans. Veh. Technol. 2021, 1-12. [CrossRef]

104. Sedjelmaci, H.; Boudguiga, A.; Ben, I.; Mohammed, S. Ad Hoc Networks An efficient cyber defense framework for UAV-Edge computing network. Ad Hoc Netw. 2019, 94, 101970. [CrossRef]

105. Tian, Y.; Yuan, J.; Song, H. Journal of Information Security and Applications Efficient privacy-preserving authentication framework for e dge-assiste d Internet of Drones. J. Inf. Secur. Appl. 2019, 48, 102354. [CrossRef]

106. Wang, G.; Yu, X.; Xu, F.; Cai, J. Task offloading and resource allocation for UAV-assisted mobile edge computing with imperfect channel estimation over Rician fading channels. EURASIP J. Wirel. Commun. Netw. 2020, 8, 1-19. [CrossRef]

107. Messous, M.; Arfaoui, A.; Alioua, A.; Senouci, S.; Ea, D.; Comté, F. A Sequential Game Approach for Computation-Offloading in an UAV Network. In Proceedings of the GLOBECOM 2017-2017 IEEE Global Communications Conference, Singapore, 4-8 December 2017; pp. 1-7. [CrossRef]

108. Li, J.; Liu, Q.; Wu, P.; Shu, F.; Jin, S. Task Offloading for UAV-based Mobile Edge Computing via Deep Reinforcement Learning. In Proceedings of the 2018 IEEE/CIC International Conference on Communications, Beijing, China, 16-18 August 2018 ; pp. 798-802. [CrossRef]

109. Ren, Y.; Xie, Z.; Ding, Z.; Sun, X.; Xia, J.; Tian, Y. Computation offloading game in multiple unmanned aerial vehicle-enabled mobile edge computing networks. IET Commun. 2021, 15, 1392-1401. [CrossRef]

110. Zhang, L.; Zhao, Z.; Wu, Q.; Zhao, H.; Xu, H.; Wu, X. Energy-Aware Dynamic Resource Allocation in UAV Assisted Mobile Edge Computing Over Social Internet of Vehicles. IEEE Access 2018, 6, 56700-56715. [CrossRef]

111. Wang, X.; Han, Y.; Leung, V.C.M.; Niyato, D.; Yan, X.; Chen, X. Convergence of Edge Computing and Deep Learning: A Comprehensive Survey. IEEE Commun. Surv. Tutor. 2020, 22, 869-904. [CrossRef]

112. Wang, H.; Ke, H.; Sun, W. Unmanned-aerial-vehicle-assisted computation offloading for mobile edge computing based on deep reinforcement learning. IEEE Access 2020, 8, 180784-180798. [CrossRef]

113. Ullah, Z.; Al-Turjman, F.; Moatasim, U.; Mostarda, L.; Gagliardi, R. UAVs joint optimization problems and machine learning to improve the $5 \mathrm{G}$ and Beyond communication. Comput. Netw. 2020, 182, 107478. [CrossRef]

114. Pourghasemian, M.; Abedi, M.R.; Salarhosseini, S.; Mokari, N.; Javan, M.R.; Jorswieck, E.A. AI-Based and Mobility-Aware Energy Efficient Resource Allocation and Trajectory Design for NFV Enabled Aerial Networks. arXiv 2021, arXiv:2105.10282. Available online: https: / / arxiv.org/abs/2105.10282v1 (accessed on 12 November 2021).

115. Xu, J.; Chen, L.; Ren, S. Online Learning for Offloading and Autoscaling in Energy Harvesting Mobile Edge Computing. IEEE Trans. Cogn. Commun. Netw. 2017, 3, 361-373. [CrossRef]

116. Wang, J.; Hu, J.; Min, G.; Zhan, W.; Ni, Q.; Georgalas, N. Computation Offloading in Multi-Access Edge Computing Using a Deep Sequential Model Based on Reinforcement Learning. IEEE Commun. Mag. 2019, 57, 64-69. [CrossRef] 
117. Bozorgchenani, A.; Member, S.; Mashhadi, F.; Member, S. Multi-Objective Computation Sharing in Energy and Delay Constrained Mobile Edge Computing Environments. IEEE Trans. Mob. Comput. 2020, 20, 2992-3005. [CrossRef]

118. Tang, L.; Tang, B.; Kang, L.; Zhang, L. A Novel Task Caching and Migration Strategy in Multi-Access Edge Computing Based on the Genetic Algorithm. Futur. Internet 2019, 11, 181. [CrossRef]

119. Dinh, T.Q.; Member, S.; La, Q.D.; Quek, T.Q.S. Distributed Learning for Computation Offloading in Mobile Edge Computing. IEEE Trans. Commun. 2018, 66, 6353-6367. [CrossRef]

120. Miao, Y.; Wu, G.; Li, M.; Ghoneim, A.; Al-rakhami, M. Intelligent task prediction and computation offloading based on mobileedge cloud computing. Futur. Gener. Comput. Syst. 2020, 102, 925-931. [CrossRef]

121. Li, G.; Zhou, H.; Feng, B.; Li, G.; Li, T.; Xu, Q.; Quan, W. Fuzzy Theory Based Security Service Chaining for Sustainable Mobile-Edge Computing. Mob. Inf. Syst. 2017, 2017, 8098394. [CrossRef]

122. Vimal, S.; Khari, M.; Dey, N.; González, R.; Robinson, Y.H. Enhanced resource allocation in mobile edge computing using reinforcement learning based MOACO algorithm for IIOT. Comput. Commun. 2020, 151, 355-364. [CrossRef]

123. Li, J.; Gao, H.; Lv, T.; Lu, Y. Deep reinforcement learning based computation offloading and resource allocation for MEC. In Proceedings of the 2018 IEEE Wireless Communications and Networking Conference (WCNC), Barcelona, Spain, 15-18 April 2018; pp. 1-6. [CrossRef]

124. Wang, J.; Member, S.; Zhao, L.; Member, S.; Liu, J.; Member, S. Smart Resource Allocation for Mobile Edge Computing: A Deep Reinforcement Learning Approach. IEEE Trans. Emerg. Top. Comput. 2019, 9, 1529-1541. [CrossRef]

125. Zhang, Q.; Lin, M.; Yang, L.T.; Chen, Z.; Li, P. Energy-Efficient Scheduling for Real-Time Systems Based on Deep Q-Learning Model. IEEE Trans. Sustain. Comput. 2017, 4, 132-141. [CrossRef]

126. Luo, Z.; Liwang, M.; Lin, Z.; Huang, L.; Du, X. Energy-Efficient Caching for Mobile Edge Computing in 5G Networks. Appl. Sci. 2017, 7, 557. [CrossRef]

127. Xiao, L.; Wan, X.; Dai, C.; Du, X.; Chen, X.; Guizani, M. Security in Mobile Edge Caching with Reinforcement Learning. IEEE Wirel. Commun. 2018, 25, 116-122. [CrossRef]

128. Huang, L.; Feng, X.; Zhang, C.; Qian, L.; Wu, Y. Deep reinforcement learning-based joint task of fl oading and bandwidth allocation for multi-user mobile edge computing. Digit. Commun. Netw. 2019, 5, 10-17. [CrossRef]

129. Li, Z. Genetic Algorithm-Based Optimization of Offloading and Resource Allocation in Mobile-Edge Computing. Information 2020, 11, 83. [CrossRef]

130. Huang, L.; Feng, X.; Feng, A.; Huang, Y.; Qian, L.P. Distributed Deep Learning-based Offloading for Mobile Edge Computing Distributed Deep Learning-based Offloading for Mobile Edge. Mob. Netw. Appl. 2018, 1-8. [CrossRef]

131. Guo, F.; Zhang, H.; Ji, H.; Li, X.; Leung, V.C.M. An efficient computation offloading management scheme in the densely deployed small cell networks with mobile edge computing. IEEE/ACM Trans. Netw. 2018, 26, 2651-2664. [CrossRef]

132. Wang, L.; Wang, K.; Pan, C.; Xu, W.; Aslam, N.; Hanzo, L. Multi-Agent Deep Reinforcement Learning-Based Trajectory Planning for Multi-UAV Assisted Mobile Edge Computing. IEEE Trans. Cogn. Commun. Netw. 2021, 7, 73-84. [CrossRef]

133. Liu, Y.; Member, S.; Chen, Y.; Member, S. Artificial Intelligence Driven UAV-NOMA-MEC in Next Generation Wireless Networks. arXiv 2021, arXiv:2101.11681. Available online: https://arxiv.org/abs/2101.11681v1 (accessed on 12 November 2021).

134. Yu, S.; Wang, X.; Langar, R. Computation offloading for mobile edge computing: A deep learning approach. In Proceedings of the IEEE International Symposium on Personal, Indoor and Mobile Radio Communications, Bologna, Italy, 9-12 September 2018; pp. 1-6. [CrossRef]

135. Chen, L.; Zhao, R.; He, K.; Zhao, Z.; Fan, L. Intelligent ubiquitous computing for future UAV-enabled MEC network systems. Cluster Comput. 2021. [CrossRef]

136. Liu, Y.; Xie, S.; Zhang, Y. Cooperative Offloading and Resource Management for UAV-Enabled Mobile Edge Computing in Power IoT System. IEEE Trans. Veh. Technol. 2020, 69, 12229-12239. [CrossRef]

137. Liu, X.; Liu, Y.; Chen, Y.; Hanzo, L. Trajectory design and power control for multi-UAV assisted wireless networks: A machine learning approach. IEEE Trans. Veh. Technol. 2019, 68, 7957-7969. [CrossRef]

138. Ma, X.; Yin, C.; Liu, X. Machine Learning Based Joint Offloading and Trajectory Design in UAV Based MEC System for IoT Devices. In Proceedings of the 2020 IEEE 6th International Conference on Computer and Communications (ICCC), Chengdu, China, 11-14 December 2020; pp. 902-909. [CrossRef]

139. Grasso, C.; Raftopoulos, R.; Schembra, G. Deep Q-Learning for Job Offloading Orchestration in a Fleet of MEC UAVs in 5G Environments. In Proceedings of the 2021 IEEE 7th International Conference on Network Softwarization (NetSoft), Tokyo, Japan, 28 June-2 July 2021; pp. 186-190. [CrossRef]

140. Zhang, C.; Zheng, Z. Task migration for mobile edge computing using deep reinforcement learning. Futur. Gener. Comput. Syst. 2019, 96, 111-118. [CrossRef]

141. Fraga-Lamas, P.; Ramos, L.; Mondéjar-Guerra, V.; Fernández-Caramés, T.M. A review on IoT deep learning UAV systems for autonomous obstacle detection and collision avoidance. Remote Sens. 2019, 11, 2144. [CrossRef]

142. Lagkas, T.; Argyriou, V.; Bibi, S.; Sarigiannidis, P. UAV IoT framework views and challenges: Towards protecting drones as "things". Sensors 2018, 18, 4015. [CrossRef]

143. Jensen, I.J.; Selvaraj, D.F.; Ranganathan, P. Blockchain technology for networked swarms of unmanned aerial vehicles (UAVs). In Proceedings of the 2019 IEEE 20th International Symposium on A World of Wireless, Mobile and Multimedia Networks (WoWMoM), Washington, DC, USA, 10-12 June 2019. [CrossRef] 
144. Stöcker, C.; Bennett, R.; Nex, F.; Gerke, M.; Zevenbergen, J. Review of the Current State of UAV Regulations. Remote Sens. 2017, 9 , 459. [CrossRef]

145. Zhang, Q.; Chen, J.; Ji, L.; Feng, Z.; Han, Z.; Chen, Z. Response Delay Optimization in Mobile Edge Computing Enabled UAV Swarm. IEEE Trans. Veh. Technol. 2020, 69, 3280-3295. [CrossRef]

146. Zhou, F.; Hu, R.Q.; Li, Z.; Wang, Y. Mobile edge computing in unmanned aerial vehicle networks. IEEE Wirel. Commun. 2020, 27, 140-146. [CrossRef]

147. Tang, S.; Zhou, W.; Chen, L.; Lai, L.; Xia, J.; Fan, L. Battery-constrained federated edge learning in UAV-enabled IoT for B5G/6G networks. Phys. Commun. 2021, 47, 101381. [CrossRef] 